\title{
In silico Design of Porous Polymer Networks: High-Throughput Screening for Methane Storage Materials
}

\author{
Richard L. Martin, ${ }^{\dagger}$ Cory M. Simon, ${ }^{\ddagger}$ Berend Smit, ${ }^{\ddagger}$ and Maciej Haranczyk ${ }^{*}{ }^{\dagger}$ \\ ${ }^{\dagger}$ Computational Research Division, Lawrence Berkeley National Laboratory, Berkeley, California 94720, United States \\ ${ }^{\ddagger}$ Department of Chemical and Biomolecular Engineering, University of California at Berkeley, Berkeley, California 94720 , United \\ States
}

Supporting Information

ABSTRACT: Porous polymer networks (PPNs) are a class of advanced porous materials that combine the advantages of cheap and stable polymers with the high surface areas and tunable chemistry of metal-organic frameworks. They are of particular interest for gas separation or storage applications, for instance, as methane adsorbents for a vehicular natural gas tank or other portable applications. PPNs are self-assembled from distinct building units; here, we utilize commercially available chemical fragments and two experimentally known synthetic routes to design in silico a large database of synthetically realistic PPN materials. All structures from our database of 18,000 materials have been relaxed with semi-

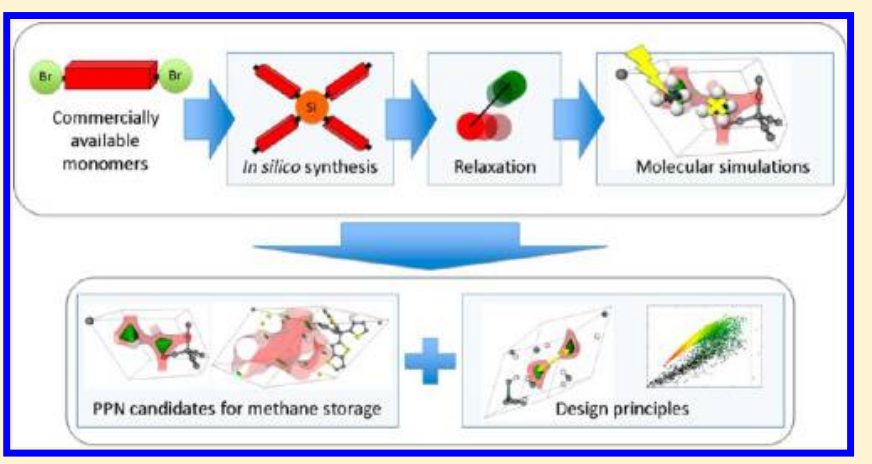
empirical electronic structure methods and characterized with Grand-canonical Monte Carlo simulations for methane uptake and deliverable (working) capacity. A number of novel structure-property relationships that govern methane storage performance were identified. The relationships are translated into experimental guidelines to realize the ideal PPN structure. We found that cooperative methane-methane attractions were present in all of the best-performing materials, highlighting the importance of guest interaction in the design of optimal materials for methane storage.

\section{INTRODUCTION}

Porous polymer networks (PPNs) are quasi-ordered porous organic polymers; a class of so-called advanced porous materials, ${ }^{1}$ PPNs combine the advantages of cheap, lightweight, and stable polymers with those of ultrahigh surface area and controllable surface chemistry metal-organic frameworks (MOFs). Since PPNs are, like other advanced porous materials, assembled modularly from building blocks, they are highly tunable materials; substitution or functionalization of building blocks can lead to a potentially unlimited space of possible materials, which can be tailored for specific applications. ${ }^{1-3}$ In particular, PPNs and related materials show significant potential for energy-related applications such as gas separation (e.g., carbon dioxide capture $)^{4,5}$ and gas storage.

PPNs (e.g., Figure 1) are synthesized through fast irreversible reactions, typically leading to amorphous powders. Computational modeling of amorphous materials is challenging due to limited experimental information on their structure. The experimental data on the structures of PPN materials typically comprise only basic characterization of density, surface area, and gas adsorption measurement. ${ }^{6}$ Therefore, unlike crystalline solids, one cannot use experimental crystal structure information as the starting point of a computational study and must rely instead upon additional assumptions regarding the structure of the material, which can at present be, at best, only indirectly validated with experiments. Despite these intrinsic difficulties, there are several examples of models with various degrees of amorphism ${ }^{6,7}$ that successfully reproduce the experimentally measured methane adsorption behavior of PPN4 , including our own work ${ }^{8}$ which assumes a crystalline model. Ideally, one could design an ordered material structure that exhibits the optimal characteristics for a given application and realize this structure experimentally. The computationally predicted structures utilized herein represent this ideal case and are based on crystalline models; as such, these models can be considered as upper bounds on the structural order or quality of these materials. We note that amorphous structural arrangements may conspire to exhibit improved application performance compared to their crystalline counterparts. However, by their amorphous nature, this performance is not foreseeable by design, and is likely variable from experiment to experiment. The crystalline assumption thus provides a standardized metric for reporting material properties.

Most experimental research on PPNs and other advanced porous materials focuses on identifying the optimal material building blocks for a particular application. The fact that advanced porous material structures are highly tunable makes them very attractive targets for such a rational design approach. In practice however, given the breadth of possibilities for

Received: December 5, 2013

Published: March 10, 2014 

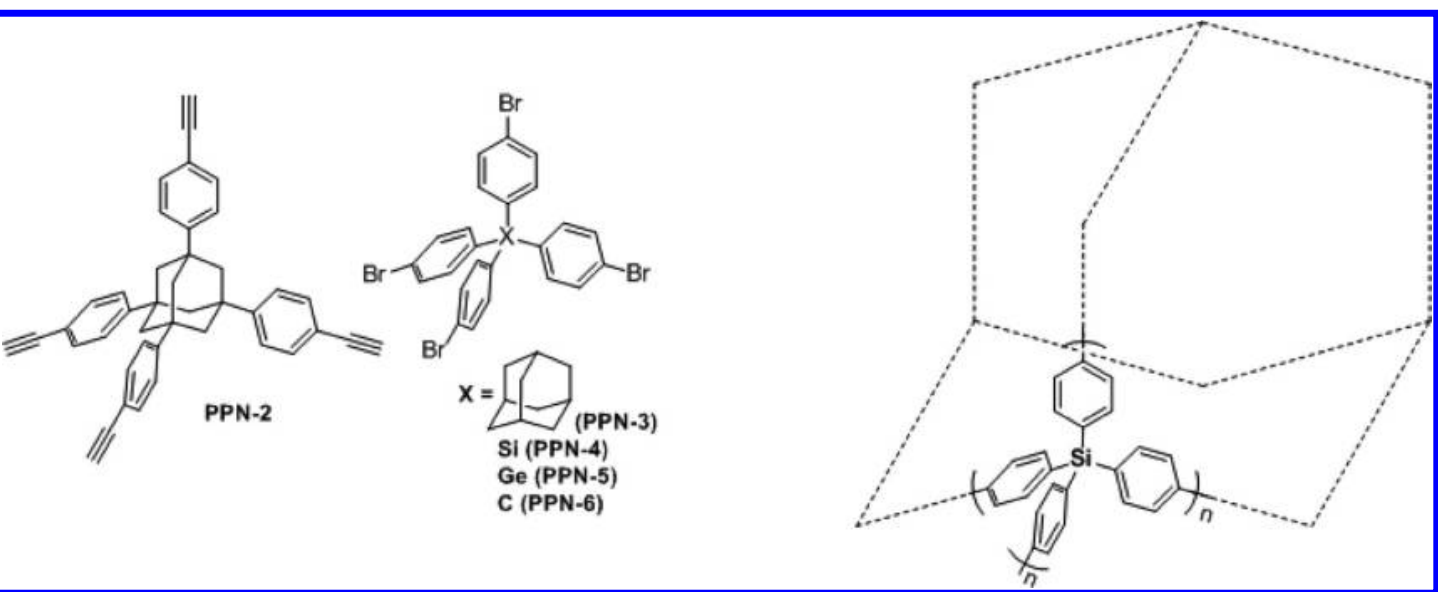

Figure 1. Left: the tetrahedral monomers of PPN-2 through PPN-6. Right: the dia-net polymeric structure of these materials illustrated using PPN-4.

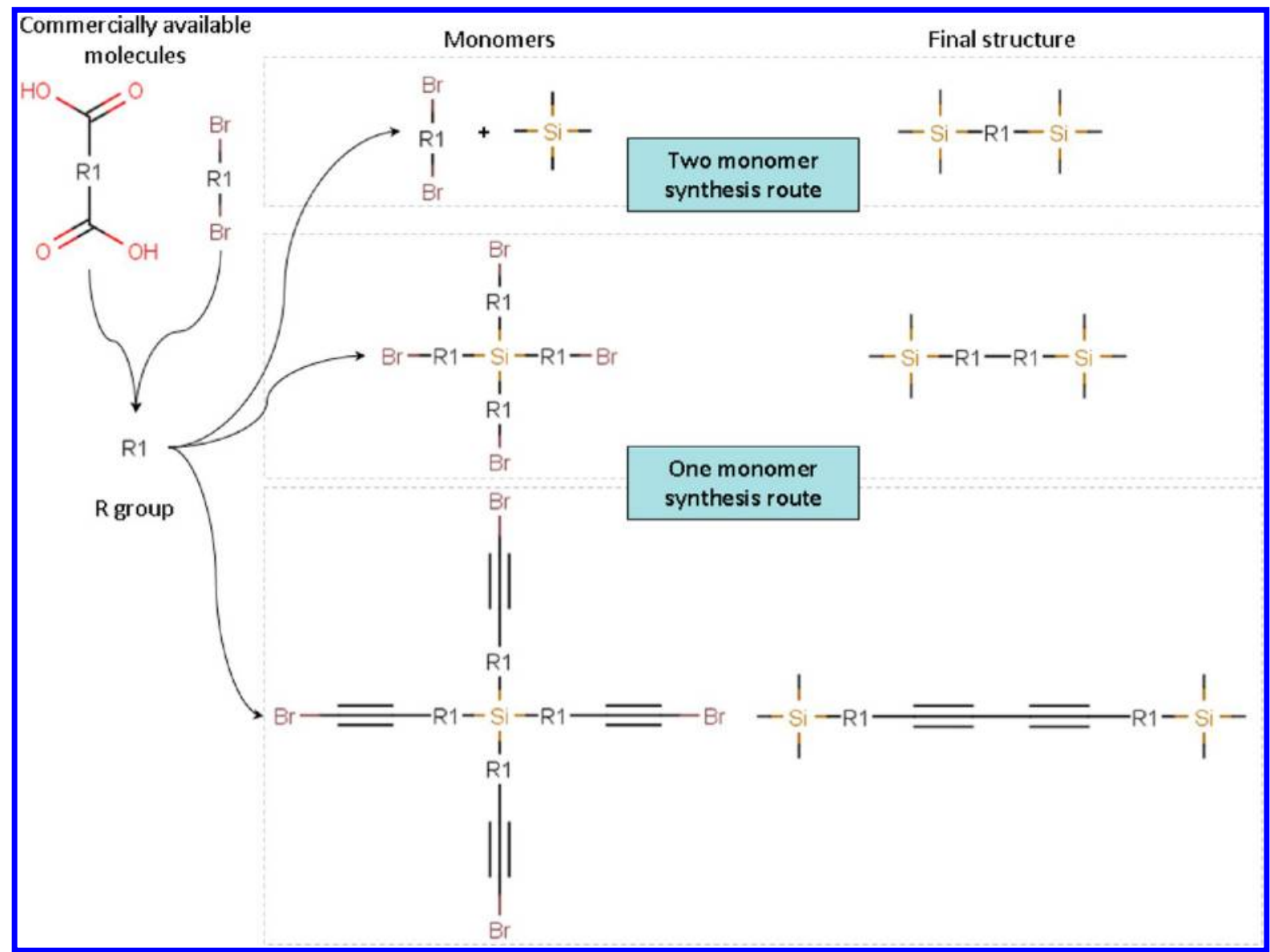

Figure 2. PPN synthesis routes resulting in $-A-B-A-$ diamond-like polymeric structures, illustrated for the silicon $A$ component. (Left) $R$ groups within commercially available dibromide and dicarboxylic organic molecules are identified. (Center) These $R$ groups are utilized to design appropriate monomers for known synthesis routes. ${ }^{17,19}$ (Right) Polymeric structure models with differing $B$-component chemistries are assembled on the basis of these monomers.

materials design and the resources (including time) required for synthesis and measurement, only a very small fraction of possible materials can be tested for a given application. In silico screening of possible PPN materials would support experimental research efforts by rapidly identifying both individual high-performance materials and design rules for achieving improved properties, enabling experimental efforts to focus on the most promising materials. An important aspect of this highthroughput screening protocol is the construction of a large data set of predicted or hypothetical PPN materials, which can be exhaustively tested computationally for application-specific performance.

In this work, we introduce such a data set of hypothetical PPN materials. These materials are analogues of the predicted structures of experimentally realized PPNs (Figure 1) and interpenetrated versions thereof. Our data set comprises 17,846 predicted diamond-like materials (i.e., exhibiting the diamond net, $\operatorname{RCSR}^{9}$ symbol dia), which are based on commercially 


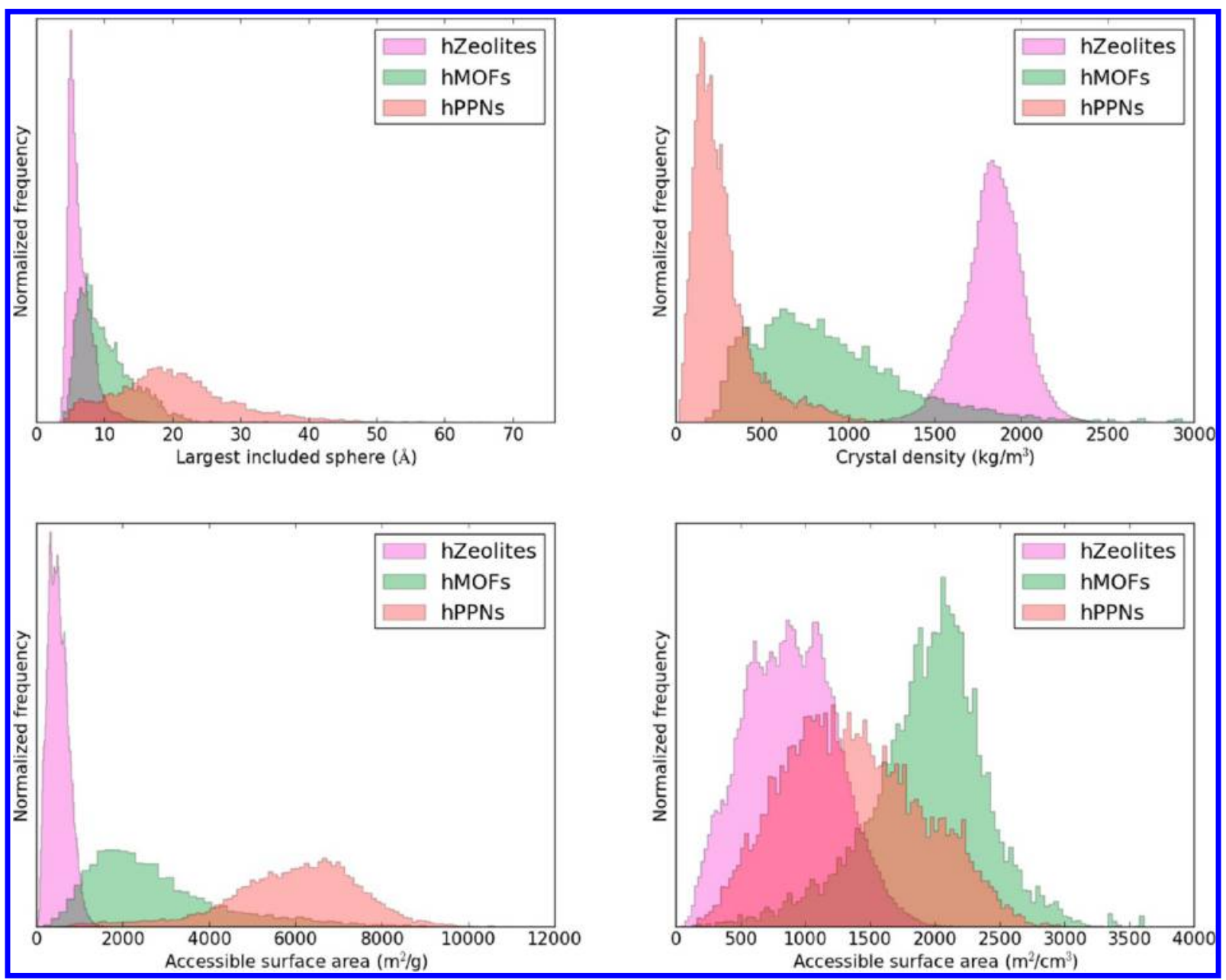

Figure 3. Histograms illustrating the distribution of geometric properties in the hypothetical PPN (hPPN) data set: largest included sphere (top left), crystal density (top right), gravimetric surface area (bottom left) and volumetric surface area (bottom right). To provide a frame of reference and to facilitate comparisons, the geometric properties of $136,328^{49}$ methane-accessible hypothetical zeolites (hZeolites) ${ }^{11,12}$ and a random selection of 10,000 hypothetical metal-organic frameworks (hMOFs) ${ }^{10}$ are included.

available chemical fragments and two experimentally known synthetic routes. Our aim is to build a set of hypothetical materials that have a high likelihood of being synthesized. Databases for other classes of materials, particularly MOFs ${ }^{10}$ and zeolites, ${ }^{11,12}$ have recently been reported and comprise hundreds of thousands or millions of hypothetical structures, respectively. To our knowledge, this is the first hypothetical PPN database, which furthermore comprises relaxed structure models based on known synthesis routes and linkers based on commercial chemical fragments (or precursors), making structures in our database close to synthetic realization. The generation of our database is described in detail in section 2.1 "Generating a PPN Database".

As an example application of this database, we evaluated all materials for their methane storage performance. Natural gas (typically $70-90 \%$ methane) is of great interest as a transportation fuel source ${ }^{13}$ since it releases less $\mathrm{CO}_{2}$ on an energy basis upon combustion by comparison to emissions of other fossil fuels, ${ }^{14}$ and its cost has dropped in recent years due to (a) the adoption of technologies for its recovery from shale; and (b) the discovery of new, large gas reserves. ${ }^{15}$

The transportation sector is an enormous potential market for natural gas to gain share over petroleum-based fuels. Methane has a relatively low energy density $(0.036 \mathrm{MJ} / \mathrm{L})$ at ambient conditions, approximately 1200 times lower than that of gasoline $(46.4 \mathrm{MJ} / \mathrm{L}){ }^{1}$ In order to obtain a satisfactory driving range from a single tank fill-up, a densification process is required. Although compressed natural gas ( $\mathrm{CNG})$ is presently in use in some larger vehicles, its utilization has not become widespread due to factors such as (a) infrastructure requirements: the multistage compressors required to pressurize natural gas to 200 bar are large and expensive and (b) tank requirements: CNG tanks, typically thick and heavy-walled, are both expensive and bulky, the latter necessitating their positioning in the rear of a personal vehicle, eliminating valuable space. As an alternative, advanced porous materials such as PPNs are being targeted as potential sorbents to store an energy density of methane similar to CNG, but at lower pressures (35-65 bar). Adsorbed natural gas technology (ANG) is promising because multistage compressors are not required to achieve a pressure of 65 bar, alleviating refill station infrastructure hindrances and facilitating the possibility of home-refueling. Furthermore, thinner-walled and conformable tanks can be utilized with the lower pressure rating.

Our procedure for evaluating PPN materials for vehicular methane storage is described in detail in section 3 "Evaluating PPNs for Methane Storage". Besides vehicular applications, ANG technology is envisioned in other applications such as 
train- or truck-based gas transport (e.g., virtual pipeline concept) and portable (e.g., camping) powered kitchen and heating equipment. PPNs may be an attractive family of materials for the latter applications, but storage targets have not yet been established.

\section{DESIGN OF A PPN DATABASE}

2.1. Generating a PPN Database. Our in silico design of PPN materials consists of the following steps. For each combination of molecular building blocks:

- generate an initial prediction of the resulting material structure by positioning the building blocks together according to the diamond topology

- relax the resulting framework using semiempirical electronic structure methods in order to refine the structural geometry from the above starting point

- while the relaxed material's pore size is large enough, search for successively higher levels of framework interpenetration, each of which is relaxed as above

Each of these steps is described in detail below. The set of resulting structures, together with their structural descriptors outlined in section 2.2, are available in ref 16 .

2.1.1. Structure Prediction. Our computationally designed PPNs all exhibit diamond-like polymeric structures of the form $-A-B-A-$, where the tetrahedral component $A$ is either adamantane, silicon, germanium, or carbon (by analogy to PPN-3 through PPN- $6^{3}$ ) and represents the vertices of the diamond-like structure (see Figure 1 ), and $B$ is some linear bridging component. To achieve hypothetical PPN structures of this form, we mimic two known synthetic routes from the literature which result in differing $B$ components, and which we refer to henceforth as the one-monomer and two-monomer routes (Figure 2). In the two-monomer route, ${ }^{17}$ the first monomer is an organic dibromide molecule of the form $\mathrm{Br}-\mathrm{R}-\mathrm{Br}$ (where $\mathrm{R}$ is some chemical species), and the second is a source of the $A$ component (e.g., for silicon, $\mathrm{SiCl}_{4}{ }^{18}$ ); together, these building units will enable formation of an EOF-1 analogue, ${ }^{17}$ i.e. a diamond-like structure comprising polymeric chains of $-A-R-$ $A-$. By contrast, the one-monomer route ${ }^{19}$ utilizes a single tetrahedral molecule; this is the synthetic route for PPN-4, and in this route the $R$ group and $A$ component are explicitly connected in the single monomer (Figure 2). In this route, the resulting structure comprises $-A-R-R-A-$ features. By analogy to $\mathrm{PPN}-2^{2}$ (see Figure 1), we also explore a variant on this route wherein the tetrahedral monomers are terminated by alkyne groups, giving rise to polymeric chains of the form $-A-R$-alkyne-alkyne-R-A-.

Our judicious selection of $R$ group chemical species comprises a library of commercially available organobromides and equivalent dicarboxylic acids, enabling us to generate synthetically feasible structures from building blocks purchasable on the Internet (Figure 2). We note that this approach can be trivially extended to include noncommercially available or hypothetical chemical fragments. Both components of this chemical library were downloaded from the eMolecules commercial database. ${ }^{20}$ We selected bromine-terminated building blocks such as organobromides since they are common in PPN synthesis and are explicitly used as building blocks in the two-monomer route; we also utilize our existing data set of dicarboxylic acids (introduced in previous work involving the in silico design of MOF-5 analogues ${ }^{21}$ ) since these are closely related compounds. For the one-monomer route, we extract the
$R$ groups of these $\mathrm{HOOC}-\mathrm{R}-\mathrm{COOH}$ and $\mathrm{Br}-\mathrm{R}-\mathrm{Br}$ molecules and incorporate them into the required tetrahedral monomers. All materials in this work are therefore based on either tetrahedral and linear building blocks (two-monomer route), or tetrahedral building blocks alone (one-monomer route); hence, according to the experimentally known preference for the diamond (dia) net in materials comprising these components, ${ }^{22,23}$ and following our previous work, ${ }^{8}$ the underlying structures of all materials are modeled with the dia net. Through this in silico design procedure and our structure assembly algorithms, ${ }^{24}$ we achieve 17,846 PPN materials. Because of the role of terminal bromine atoms in PPN synthesis, we omitted $R$ groups containing bromine in our search, as this would introduce cross-linking and undermine our assumption of the dia net.

We note that this workflow can be applied more generally to other building-block combinations which give rise to different topologies. ${ }^{25}$ Our recent work comparing topologies of MOFs revealed that materials of the dia net, and in general, all nets comprising linear linkers, have a preference for exhibiting high gravimetric surface area at the expense of volumetric surface area. ${ }^{26}$ Hence, we postulate that, in order to achieve the high volumetric methane storage capacities envisioned by the ARPAE targets, PPNs and other materials with topologies other than dia such as those based on trigonal building blocks (e.g., pyr, which was projected to achieve the highest compromise between volumetric and gravimetric surface area ${ }^{26}$ ) must also be explored. However, at present there is to our knowledge no experimental data on PPNs based on alternative building blocks and topologies which would be required to establish an appropriate modeling approach.

2.1.2. Structural Relaxation. We relaxed the assembled structure models using the semi-empirical PM6 electronic structure method implemented in MOPAC2012. ${ }^{27,28}$ Periodic boundary conditions were used in these calculations, and the periodic unit cell parameters were also permitted to relax. To describe dispersion and hydrogen bonds, we utilized the DH2 correction to PM6. ${ }^{28,29}$ We recently verified the applicability of the PM6-DH2 method to porous polymers by comparing relaxed structures to those obtained by density functional theory (DFT, i.e. a higher level of theory, and typically a greater than 2 orders of magnitude higher computational cost). In the case of both MOF-5 analogues and PPNs, ${ }^{30}$ we observed very good agreement. We note that PM6 was observed to produce slightly smaller unit cells, leading to smaller pores and higher structural densities. For the investigated PPN structures with carbon and silicon $A$ components, all measurements were less than $2.5 \%$ removed from those predicted by DFT, and in the worst case PM6 produced a 7.5\% higher density (for a germanium-based PPN).

2.1.3. Interpenetration Handling. Network interpenetration is a phenomenon which occurs in highly porous materials, whereby two or more independent frameworks interlock. It is typically considered a negative agent ${ }^{31}$ since it leads to a reduction in pore volume and surface area and an increase in density. Interpenetrated states commonly occur since they are often energetically favorable with respect to the noninterpenetrated state; controlling or preventing interpenetration during synthesis therefore remains a considerable challenge. ${ }^{32,33}$ A key component of our systematic materials design approach is to predict the likelihood and exact mode and degree of interpenetration for reliable structure prediction and evaluation. 


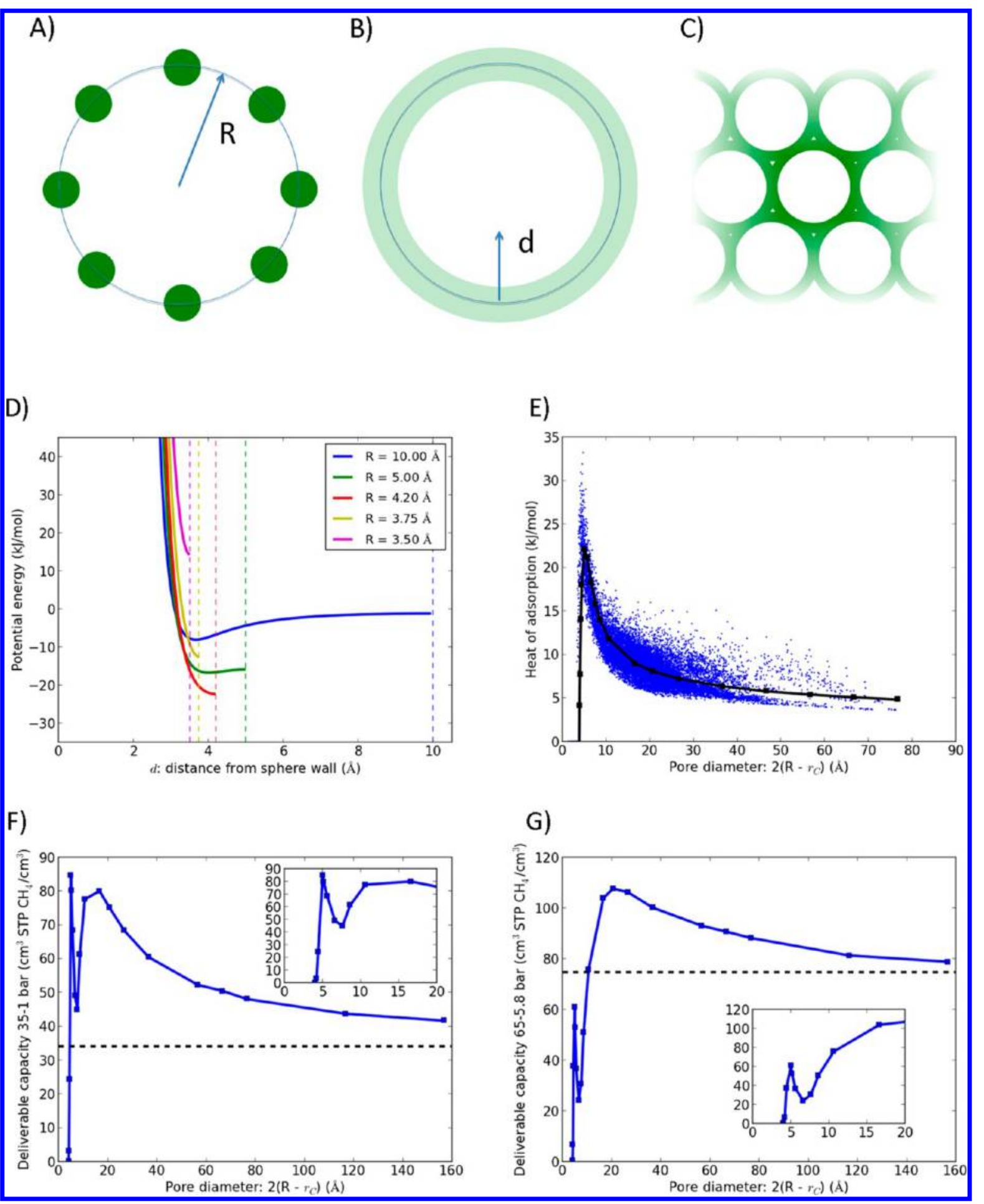

Figure 4. Spherical shell model. (A) The adsorption pocket of our model material consists of a spherical shell of framework atoms of uniform, constant surface density. (B) The potential in eq 1, a function of the distance $d$ from the shell wall, is obtained by smearing the carbon atoms over the shell with a surface integration. The unit cell is defined as the sphere of radius $R$ (blue line). (C) The wall of the sphere is shared by many adjacent unit cells; hence, the bulk material is modeled as closely packed unit cells, illustrated with a hexagonal lattice. (D) Plots of the potential energy of methane inside the pore, given in eq 1. (E) The surface density of carbon atoms in our shell was chosen to be 0.15 atoms $/ \AA^{2}$ to recapitulate the relationship between the heat of adsorption and pore diameter in the PPN data. Each PPN structure is a blue point; each model simulation is a black square. (F,G) Computed deliverable capacity of methane in the model against pore size. Dashed line is the deliverable capacity of a free space tank assuming pressure equals fugacity, as assumed in this sphere model analysis. Inset is a zoomed-in version to illustrate the two peaks in deliverable capacity.

In previous work, we observed that of all noncrystalline interpenetrated modes of PPN structure models, those with the lowest framework energy were those comprising closely interlocking nets. ${ }^{8}$ This arrangement is in stark contrast to the interpenetrated modes observed in crystalline materials, which may exhibit the most symmetric arrangements. ${ }^{34}$ As 


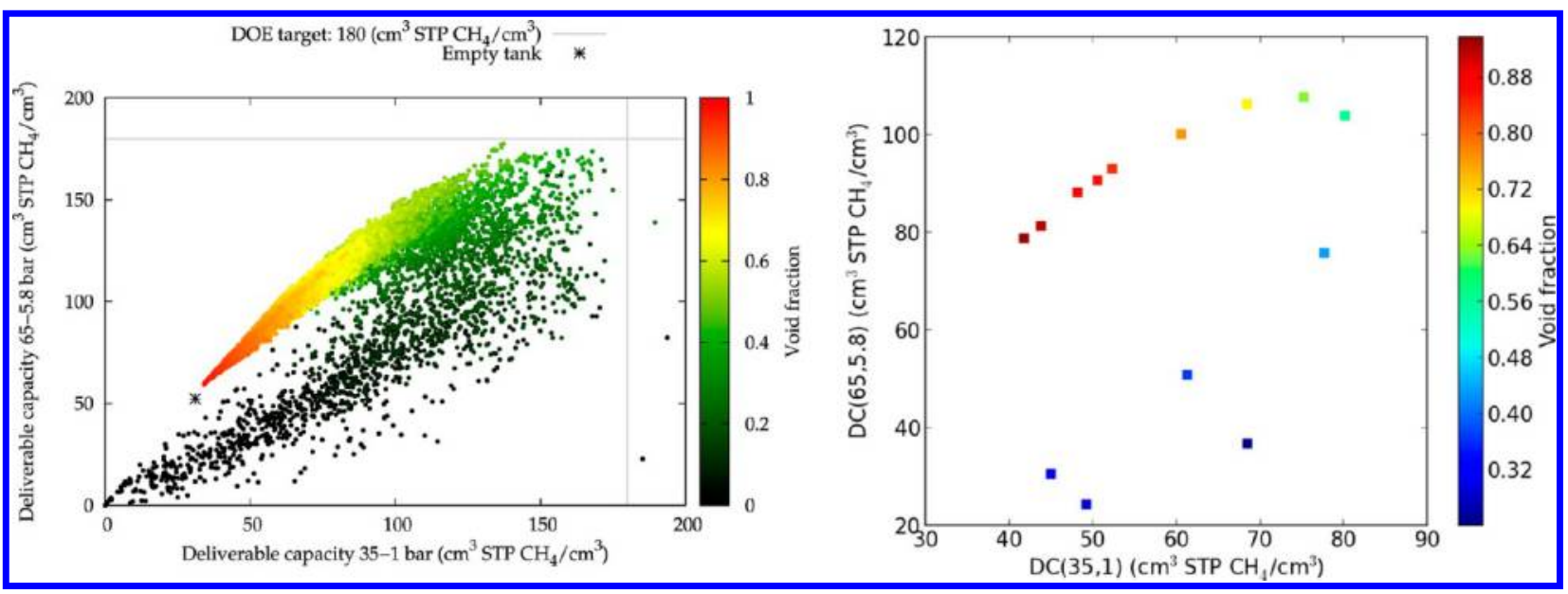

Figure 5. Performance curve illustrating DC $(65,5.8)$ versus DC $(35,1)$ for PPN structures, left, and spherical models of different radii, right (model illustrated in Figure 4). Data points are color-coded by void fraction. Gray lines indicate the DOE target of $180 \mathrm{~cm}_{\text {STP }}^{3}\left(\mathrm{CH}_{4}\right) / \mathrm{cm}^{3}$, while the cross represents the calculated deliverable capacity of an empty tank. The model captures the shape of the performance curve we observe in the PPN structures as well as the scattering at low void fractions.

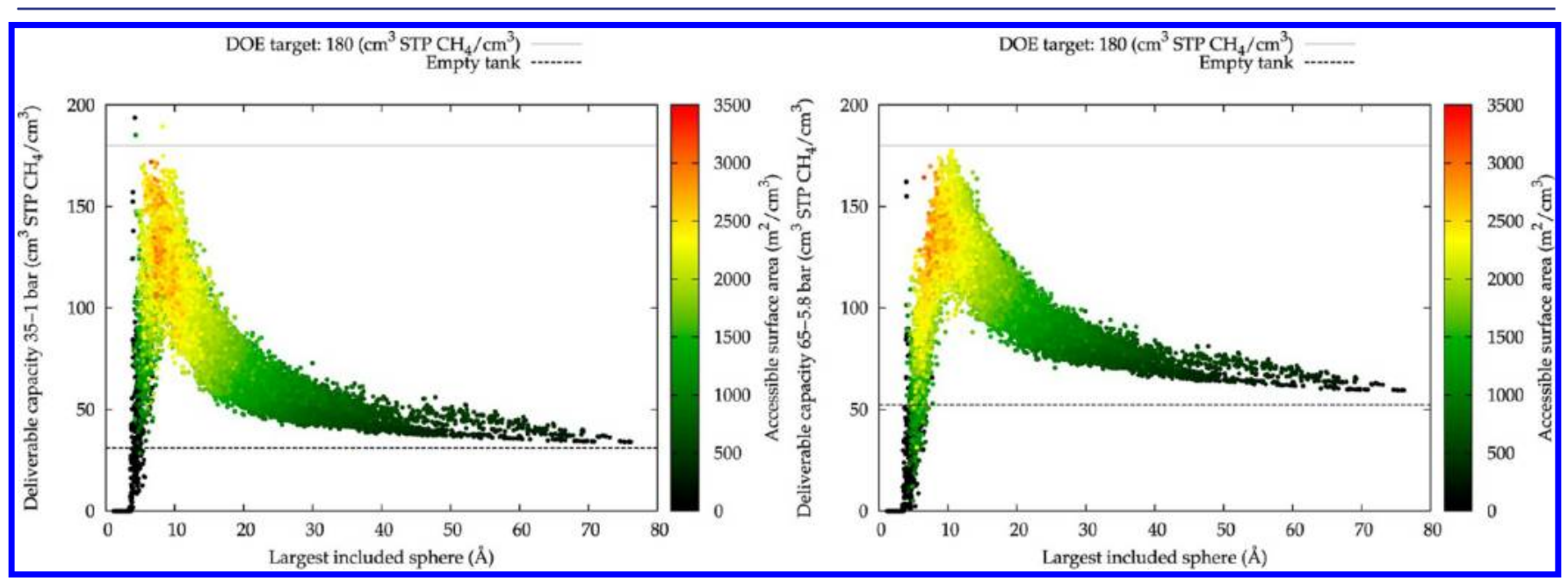

Figure 6. $\mathrm{DC}(35,1)$, left, and $\mathrm{DC}(65,5.8)$, right, as a function of the largest included sphere diameter, color-coded by volumetric surface area. This behavior is qualitatively reproduced by the sphere model (Figure $4 \mathrm{~F}, \mathrm{G}$ ).

evidence for interlocking nets in PPNs, we found that the noncrystalline interpenetrating model reproduced the experimental methane isotherms in PPNs, whereas the highly symmetric interpenetration mode could not. ${ }^{8}$ The configuration comprising closely interlocking nets is more energetically favorable due to the van der Waals interactions between the networks. This manifests in a negative correlation between exposed surface area and framework energy. We exploited this correlation to predict interpenetrated states: evaluating structural energetics is highly demanding computationally, while surface area computation (using our materials analysis software Zeo $++^{35}$ ) is comparatively inexpensive, allowing us to efficiently predict interpenetrated modes in a high-throughput manner.

The starting point for our interpenetration search is the set of relaxed, noninterpenetrated structures. For each structure, we attempt interpenetration if the diameter of the monomer is smaller than the restricting pore aperture of the material, with a $1 \AA$ tolerance. We then select the lowest surface area arrangement of nets and repeat this procedure for further levels of interpenetration until either the pore diameter is no longer large enough or until there is insufficient space to position another network. Pore size comparison is not necessarily a determinant of the likelihood of interpenetration in PPN materials; however, it is a lower bound for the potential degree of interpenetration in these materials, and our database can easily be extended to include further degrees of interpenetration. Our interpenetrated models are initial estimates of the interpenetrated state, and, like noninterpenetrated models, structural relaxation is critical. Each interpenetrated state is therefore relaxed using the same procedure described above.

2.2. Characterization of the PPN Database. Here we briefly summarize selected morphological characteristics of this PPN database, illustrated with histograms in Figure 3; to provide a frame of reference, we also compare to the properties of other hypothetical materials databases: zeolites ${ }^{11,12}$ and MOFs. ${ }^{10}$ The geometrical parameters describing the pores were calculated using our Zeo++ $\operatorname{code}^{35}$ and the set of radii provided by the Cambridge Crystallographic Data Centre (CCDC).$^{36-38}$ We note that the PPN database is the only one in which all materials exhibit a consistent topology. The PPN database can be seen to comprise materials with a wide range of pore sizes; the use of the dia net in PPN generation leads to very large 


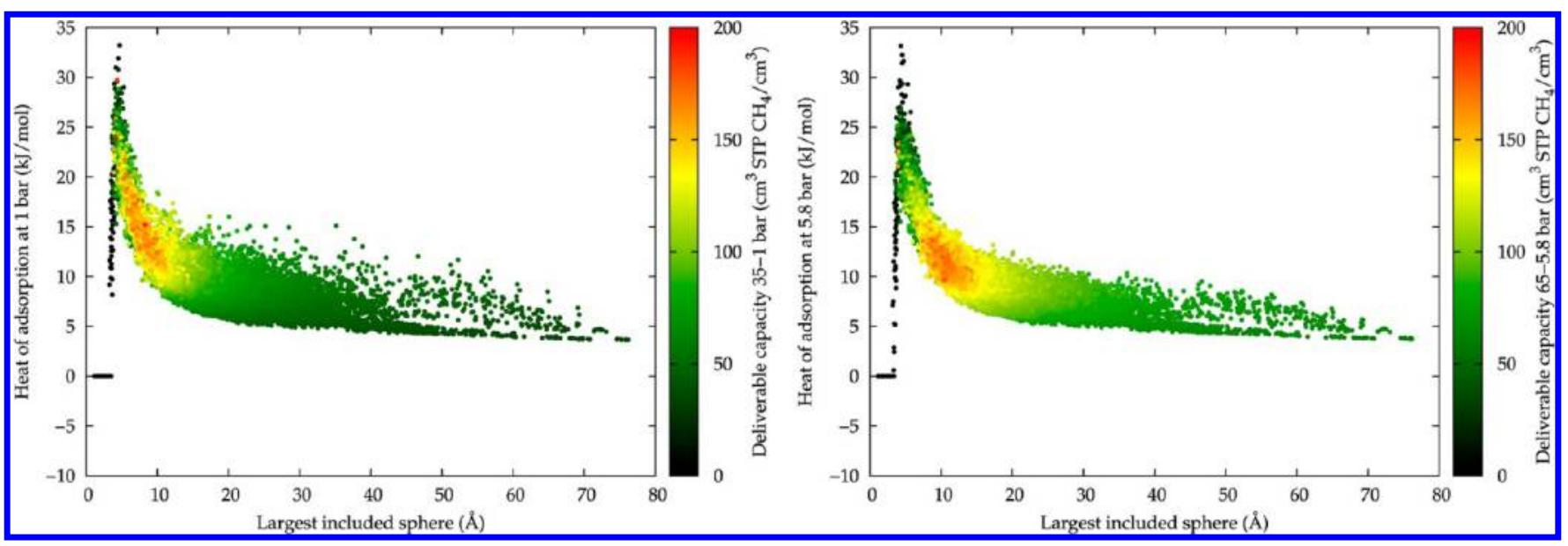

Figure 7. Heat of adsorption at depletion pressure as a function of largest pore diameter, illustrating that pore diameter is, to a degree, a proxy for heat of adsorption. Points are color-coded by deliverable capacity, illustrating where the best performing materials lie on this curve. Left: DC $(35,1)$; right: $\mathrm{D}(65,5.8)$. This behavior is qualitatively reproduced by the sphere model (Figure 4E).

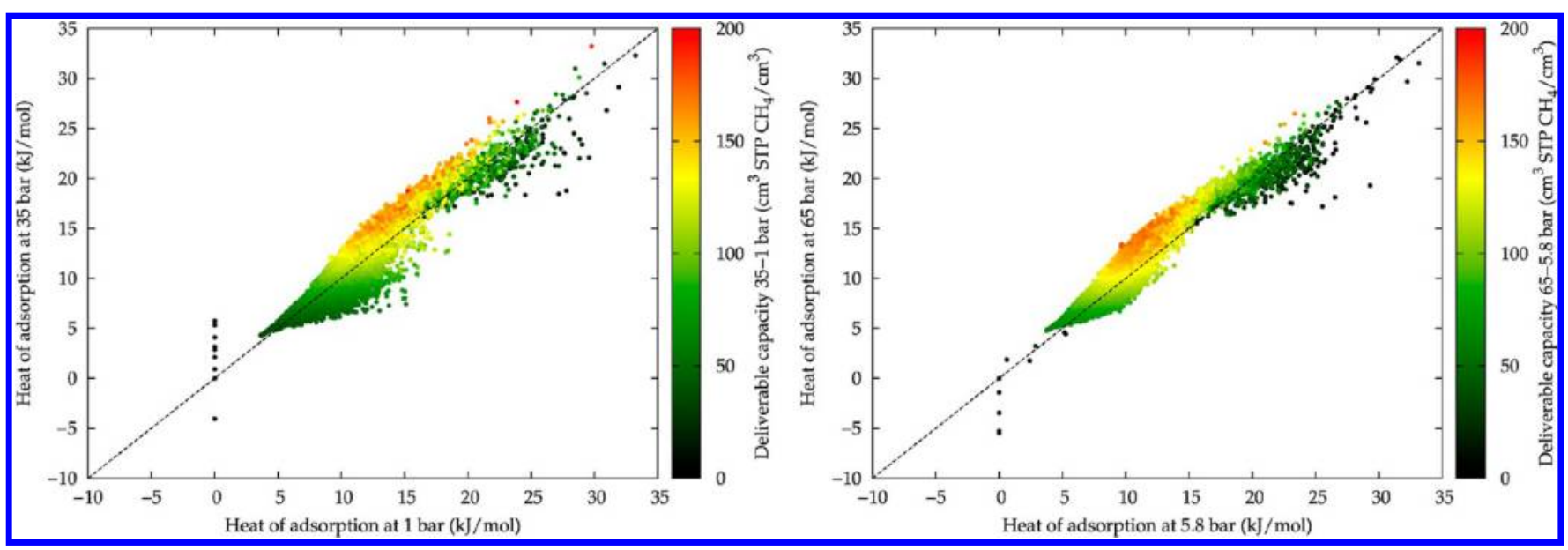

Figure 8. Heat of adsorption at the tank charging pressure against that at the tank discharging pressure for $\mathrm{DC}(35,1)$, left, and $\mathrm{DC}(65,5.8)$, right, color-coded by deliverable capacity. The structures with the highest increase in heat of adsorption with loading produce the largest deliverable capacities. The dashed line represents equal heat of adsorption at charging and discharge pressures.

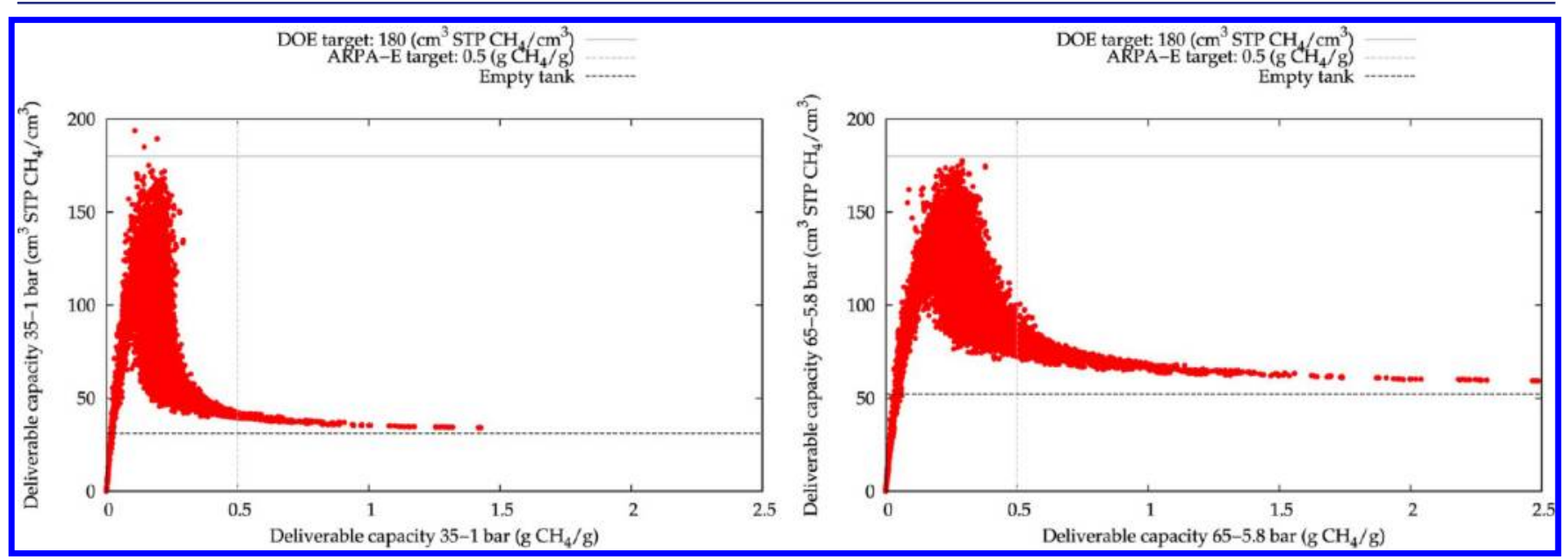

Figure 9. Gravimetric versus volumetric DC(35,1), left, and DC $(65,5.8)$, right. These two objectives can be seen to compete. The highest gravimetric deliverable capacity is trivially observed in empty tank-like materials (dashed line represents empty tank performance). Solid lines represent the relevant ARPA-E and DOE targets.

pore structures when large bridging molecules are utilized, with the largest pore diameter observed being 76.2 Å. Figure 3 illustrates that these large pores are not observed in zeolites, which have a strong tendency toward small pores. While MOFs do exhibit large pores, their median diameter is closer to that of zeolites. As a consequence, the crystal densities of PPNs can be 
Table 1. Six Highest DC $(35,1)$ Materials Identified for the Two-Monomer Synthesis Route

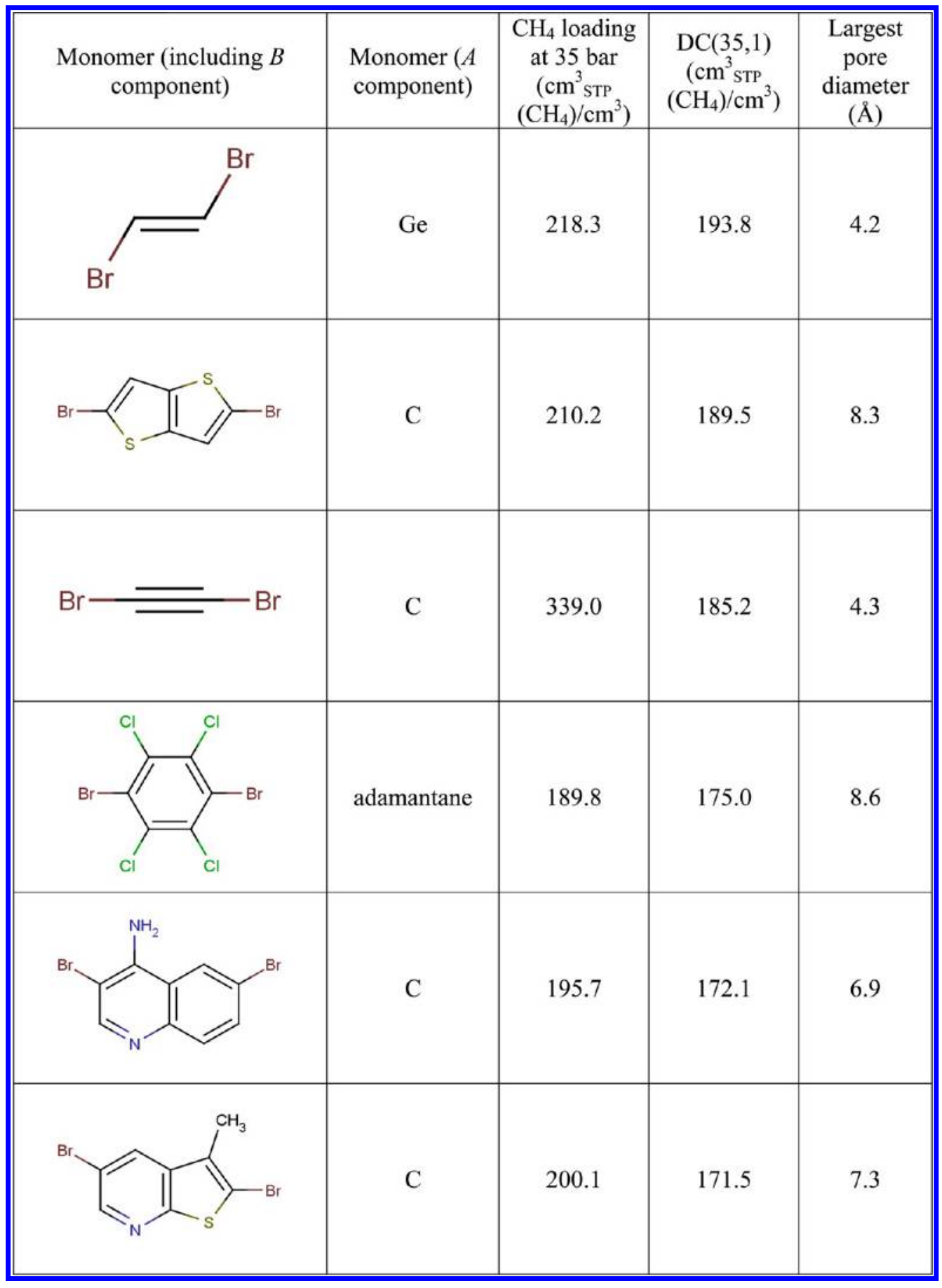

seen to be, on average, very low, and gravimetric surface areas to be high compared to both other material classes. Finally, the distribution of volumetric surface areas (VSA) of PPNs can be seen to lie approximately between those of zeolites and MOFs. The diversity of pore sizes in our PPN database holds promise for finding future applications other than methane storage.

\section{EVALUATING PPNS FOR METHANE STORAGE}

To illustrate one use of our database of PPN materials, we have screened all materials as candidates for methane storage in vehicular gas tanks. These calculations consist of predicting each material's equilibrium methane uptake using Grandcanonical Monte Carlo simulations. Before we describe the details of these calculations, we first introduce the metric by which we rank the performance of materials.

3.1. Metrics, Methods, and Computational Details. 3.1.1. Performance Metric. The primary screening criterion for evaluating methane storage materials is the deliverable (or working) capacity, i.e., the amount of methane that can be retrieved from a fully charged ANG tank. Deliverable capacity is therefore calculated as the difference in methane loading at the pressure of a filled tank and at that of a depleted tank.

In 2012, ARPA-E, the U.S. Department of Energy (DOE) subsidiary, established deliverable capacity targets for vehicular methane storage performance (ARPA-E MOVE program ${ }^{39}$ ). At the tank-filling pressure of 35 bar (or 65 bar) and desorption 


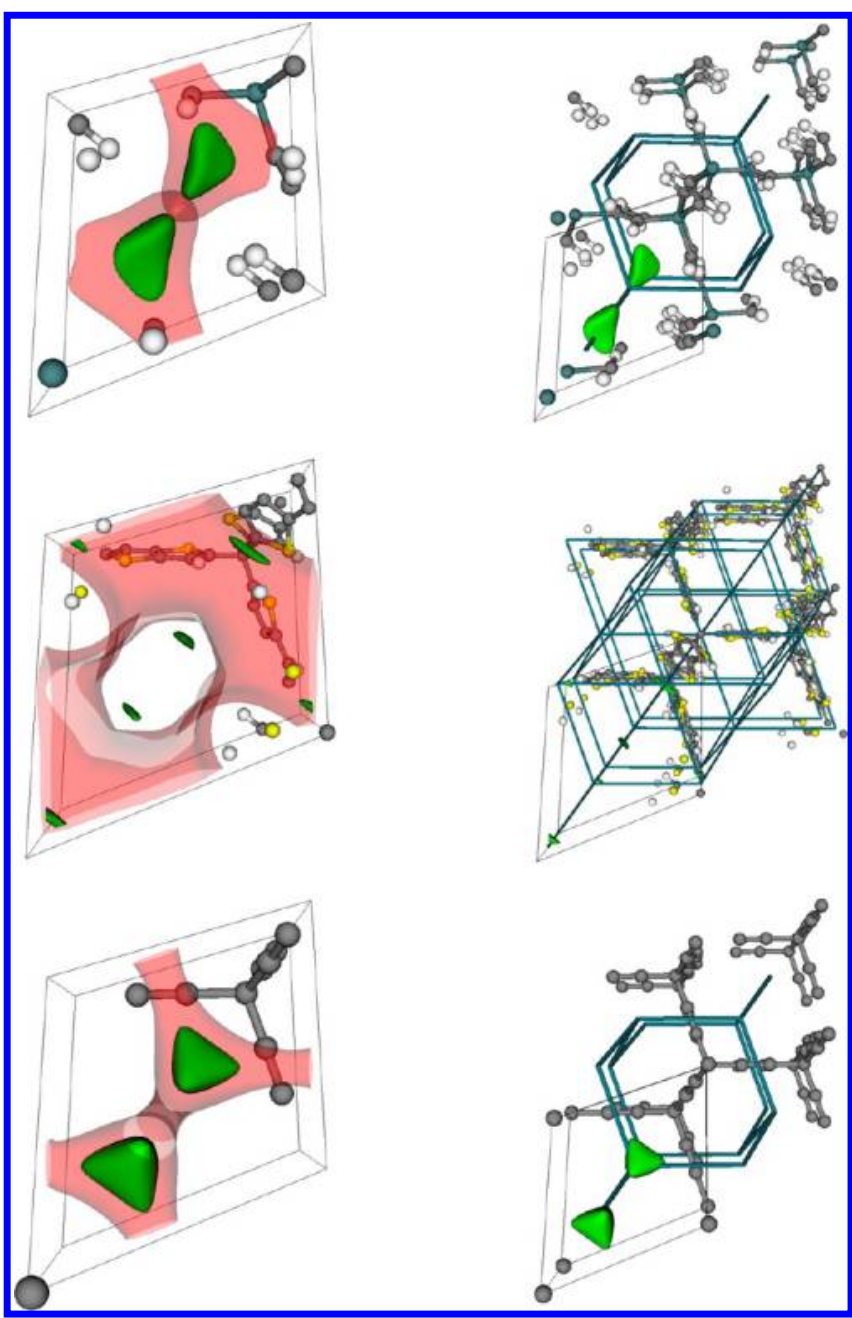

Figure 10. (Top, middle, bottom) In decreasing deliverable capacity order, PPNs A, B and C which exhibit DC $(35,1)$ greater than 180 $\mathrm{cm}^{3}$ Бтр $\left(\mathrm{CH}_{4}\right) / \mathrm{cm}^{3}$. (Left) Each material's free energy landscape. The red surface denotes energy of $37.17 \mathrm{~kJ} / \mathrm{mol}$, i.e. $15 \mathrm{RT}$ (where $\mathrm{R}$ is the universal gas constant and $T=298 \mathrm{~K}$ ), and approximates the boundary of the accessible pore space, while the green surface denotes energy of $-16.63 \mathrm{~kJ} / \mathrm{mol}$, and illustrates the strongest guest-host binding sites. (Right) The distribution of energy minima in each material can be seen to exhibit specific topological arrangements; above and below, the lowest energy positions exhibit the dia net, while in center, the bcu net.

pressure of 1 bar (or 5.8 bar) the sorbent material should exhibit a volumetric deliverable capacity of at least $315 \mathrm{~cm}^{3}$ STP $\left(\mathrm{CH}_{4}\right) / \mathrm{cm}^{3}$ at ambient temperature $(298 \mathrm{~K})$. Furthermore, to avoid a heavy tank, a gravimetric deliverable capacity target has also been set at $0.5 \mathrm{~g}\left(\mathrm{CH}_{4}\right) / \mathrm{g}$ (sorbent). However, the volumetric deliverable capacity holds greater importance for enabling ANG technology since it dominantly determines the driving range from a tank fill-up. ${ }^{13}$ These targets build upon the previous DOE methane storage target of $180 \mathrm{~cm}_{\text {STP }}^{3}\left(\mathrm{CH}_{4}\right) /$ $\mathrm{cm}^{3}$. To date, no material has met these latest ambitious targets. However, there is at present considerable experimental and theoretical research devoted to the design and discovery of new sorbent materials to reach these targets. For consistency with ARPA-E targets, we consider the deliverable capacity in two pressure ranges: between 35 (filling pressure) and 1 bar (depletion pressure), referred to as $\mathrm{DC}(35,1)$, and between 65 and 5.8 bar, $\mathrm{DC}(65,5.8)$.

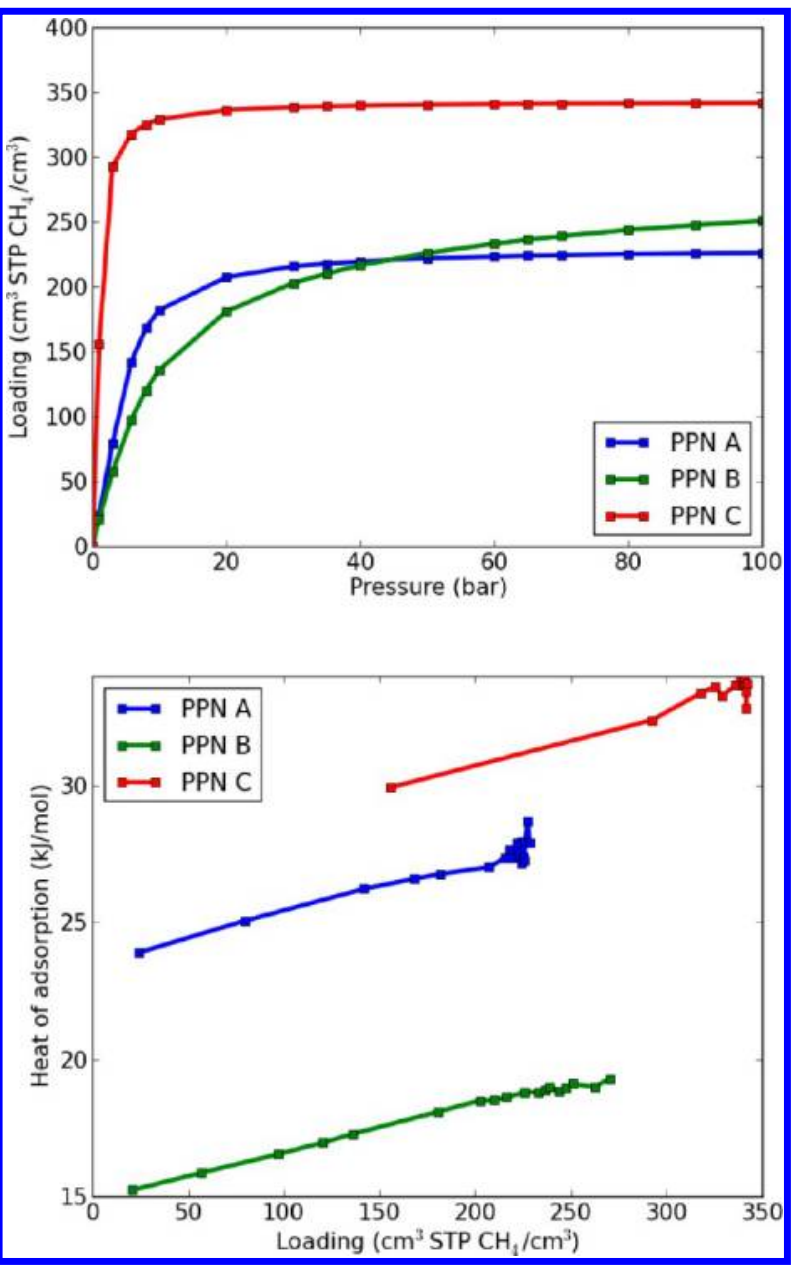

Figure 11. Adsorption isotherms (left) and heat of adsorption as a function of loading (right) for the three hypothetical materials with $\operatorname{DC}(35,1)$ above $180 \mathrm{~cm}_{\text {STP }}^{3}\left(\mathrm{CH}_{4}\right) / \mathrm{cm}^{3}$. The increasing heat of adsorption indicates the presence of methane-methane attractions.

We note that, at the point of practical application, the adsorbent in the methane storage tank will not be a perfect, single crystal. Accordingly, the deliverable capacity of the final, bulk adsorbent will necessarily be less than that of the singlecrystal; hence, our reported single-crystal deliverable capacities are an upper bound. (Note: the ARPA-E target is for a singlecrystal adsorbent, and packing losses of $25 \%$ have been anticipated in stating the target to achieve an energy density equivalent to CNG.) The final, bulk adsorbent properties depend upon the specific synthesis pathways and conditions as well as crystal packing procedures and conditions.

3.1.2. Molecular Simulation Techniques and Force Field. To predict the methane storage capacity at ambient temperature $(298 \mathrm{~K})$ and this array of pressures requires the calculation of adsorption isotherms of methane in the PPNs. Adsorption isotherms are conveniently calculated in the Grandcanonical Monte Carlo ensemble. ${ }^{40}$

In the absence of methane-specific force fields for PPNs, we use an off-the-shelf force field, Dreiding, ${ }^{41}$ to calculate guestframework interactions. We exploit the symmetry and nonpolarity of methane by modeling it using the united atom approximation and taking Lennard-Jones (LJ) parameters from the TraPPE forcefield. ${ }^{42}$ We mimic an infinite crystal structure by implementing periodic boundary conditions with a shifted LJ potential using a cutoff of $12 \AA$. LJ cross-interaction 
parameters were determined by the Lorentz-Berthelot mixing rules. ${ }^{43}$ Pressures were converted to chemical potentials using the Peng-Robinson equation of state. ${ }^{44}$ Figure S1 in the SI compares density calculations of methane at $1,5.8,35$, and 65 bar using the TraPPE force field and the Peng-Robinson equation of state with experimental measurements. ${ }^{45}$ We assume the framework to be rigid during simulation by fixing atomic positions at their relaxed crystallographic coordinates.

For the purposes of validating our modeling procedure, we compare the experimental methane adsorption of PPN-3 and PPN $-4,{ }^{3}$ as well as PPN-101, ${ }^{46}$ to their simulated uptake. We observe very good agreement for our close-net interpenetrated model of PPN-3 and non-interpenetrated model of PPN-4 (Figure S2 in the SI). We also observe good qualitative agreement for a non-interpenetrated model of PPN-101, although experimental data are at present only available at low pressure $^{46}$ (Figure S3 in the SI). At this point we have to add a note of caution, as the published structures of PPN materials depend on the synthetic procedure. ${ }^{17}$ In our comparison we have used the structure with the highest reported order, but more experimental data are required to establish the true structure of these materials. ${ }^{47}$

3.2. Simple Model Calculations. To develop an intuition on how the deliverable capacity of a material depends on its pore size, we study here a simple model of a diamond net PPN. As we shall see in the following section, the predictions made by this simple model capture, qualitatively, the relationship between methane adsorption and pore size in our data set. In this model, we assume that the adsorption pockets created by our PPN materials can be mapped to a spherical shell of uniformly distributed carbon atoms with radius $R$, as depicted in Figure 4A. The bulk material is then represented with a close packing of spheres as in Figure 4C, a modeling scheme previously proposed for analysis of PAF materials. ${ }^{48}$ Because the carbon atoms of a shell are shared between multiple adsorption pockets, we define, as an approximation, the unit cell of our material as the sphere of radius $R$. We analyzed zeolites in the context of this model in our previous work ${ }^{49}$ to capture a correlation between enthalpy and entropy change upon adsorption.

We assume that the methane-PPN interactions in our model can be described with LJ potentials. As we assume a uniform density of carbon atoms, we can effectively 'smear' each carbon atom over the shell (see Figure 4B) by surfaceintegrating the LJ potential to obtain an effective carbonmethane potential for inside the pore that is a function of the distance from the spherical shell wall, $d:{ }^{51}$

$$
\begin{aligned}
U(d ; R)= & 4 \varepsilon \frac{2 \pi R}{R-d}\left\{\frac{\sigma^{12}}{10}\left[\frac{1}{d^{10}}-\frac{1}{(2 R-d)^{10}}\right]\right. \\
& \left.-\frac{\sigma^{6}}{4}\left[\frac{1}{d^{4}}-\frac{1}{(2 R-d)^{4}}\right]\right\}
\end{aligned}
$$

where $\varepsilon$ and $\sigma$ are the LJ parameters for carbon-methane interactions taken from the Dreiding ${ }^{41}$ and TraPPE force fields. ${ }^{42}$

We can use this simple model to study the effect of changes in the pore radius $R$ on the energetics of methane inside the pore. Figure $4 \mathrm{D}$ shows the dependence of the energy on the position of a methane molecule in the pocket for a sequence of pore sizes. In the largest pores, only a thin strip around the inner-surface of the shell has a favorable potential energy for adsorption. The innermost region of these large pores is outside the range of attractive van der Waals interactions with the framework and, in a sense, is thus wasted space because the energetics are similar to that of an empty tank. As the sphere radius is reduced, the strip on the inner surface lies within the attractive region of more framework atoms in the shell, and the attractions in the strip become stronger. We begin to utilize the inner pore as well, which experiences more negative energies as it falls within the attractive regime of the framework atoms of the shell. Eventually, the energy minimum occurs in the center of the pore, where the potential overlap is maximized since methane interacts equally with all framework atoms in the shell. A global minimum of potential energy in $(R, d)$ space occurs at $(R=4.2 \AA, d=R)$. As the radius gets too small, however, repulsive forces cause the energy within the pore to become unfavorable for adsorption.

Pore size is coupled to the energetics in our model because it determines (a) the proximity of carbon atoms to the adsorbed methane in the pocket and (b) the degree of potential overlap from multiple framework atoms that can combine forces and recruit methane as guests. Using the potential in eq 1 and Widom insertions, ${ }^{40}$ we calculate the infinite dilution heat of adsorption of our model as a function of pore size, plotted in black in Figure 4E. We choose the density of carbon atoms in the spherical shell to be $0.15 \AA^{-2}$ for an appropriate mapping of our PPN structures to the spherical model; overlaid in Figure $4 \mathrm{E}$ is a scatter plot of the calculated heat of adsorption of methane at 1 bar of each structure in our PPN database against the largest included sphere calculated with Zeo++. ${ }^{35}$ To correspond to the Zeo++ calculations, the pore diameter of our model PPN is $2\left(R-r_{\mathrm{c}}\right)$, where $r_{\mathrm{c}}=1.7 \AA$ is the van der Waals radius of carbon as provided by the CCDC. ${ }^{36-38} \mathrm{We}$ achieve excellent agreement between our spherical shell model and the PPN data in both the scale of the heat of adsorption and the pore size at which repulsive forces dominate and the heat of adsorption rapidly decreases.

We expect the pore size in our model to have a strong influence upon the deliverable capacity for two reasons. First, as Figure 4E shows, the pore size controls the heat of adsorption. To obtain the highest deliverable capacity, the affinity of methane with the solid framework must be strong enough to recruit methane at the charging pressure yet weak enough to release it at the discharge pressure. ${ }^{52}$ In our previous work, we showed that there is indeed an optimal heat of adsorption but that it depends on the pore size. ${ }^{49}$ Second, the free volume in the material increases with increasing pore size because the carbon atoms in the shells take up a lesser fraction of the space occupied by the bulk material (Figure S4a in the SI).

Using Grand-canonical Monte Carlo simulations (1 million trial moves), we computed the equilibrium loading of methane in a unit cell of our model material at $298 \mathrm{~K}$ for a sequence of radii (Figure S4c,d in the SI). The deliverable capacity as a function of the pore diameter is shown in Figure 4F,G. For the smallest pores, we see a low deliverable capacity; the repulsive energy makes it unattractive for methane to adsorb, and most of the bulk material is occupied by the carbon atoms in the shell. For very large pores, the deliverable capacity approaches that of empty space (dashed line) because (a) most of the bulk material is pore volume and (b) very little of the pore volume is close enough to the shell surface to experience the attractive forces of the carbon atoms in the shell. At intermediate pore sizes, two maxima occur. The first maximum (approximately 5 $\AA$ ) occurs where the methane pore size becomes just large 
enough to fit one methane molecule. As the pore size increases beyond this point, the extra volume is, in a sense, wasted space until the pore is large enough to accommodate two methane molecules, and the (volumetric) deliverable capacity consequently drops. Figure S4b in the SI shows the loading as a function of pore size in the units of molecules per unit cell, and indeed, the described plateau in loading can be observed in the approximate pore diameter range of 5-7 $\AA$. The rise to the second peak corresponds to both an increase in free volume as the pore can accommodate more than one methane molecule and a weaker heat of adsorption that mitigates the low-pressure loading. The fall of the second peak occurs because, despite the increase in free volume with larger pore sizes, the affinity with methane becomes very weak as the pore size becomes too large. Space in the center of very large pores is too far from the carbon atoms for favorable interaction, and the loadings/ deliverable capacity approach that of the bulk methane gas in the pore volume (Figure S4 in the SI).

Because our PPNs are consistently of dia topology and explore a vast space of pore sizes, we expect the pore diameter to play a central role in determining the deliverable capacity through its link with the heat of adsorption and pore volume available for methane occupancy.

3.3. Screening Results. Our high-throughput screening protocol comprises computing the adsorption isotherms for all hypothetical PPNs. Data on such a large number of structures allow us to identify some important general characteristics of these materials.

3.3.1. Performance. In Figure 5 we summarize our screening of the entire database. This figure shows $\mathrm{DC}(65,5.8)$ and $\mathrm{DC}(35,1)$ for all materials, colored by material void fraction (i.e., volume of void space per volume of material). As a reference, we also plot the deliverable capacity of a free-space tank (denoted by a cross) to illustrate the gain in performance offered by adsorbed natural gas technology. As expected, those materials with the largest void fraction have a performance that is not much better than a free-space tank. For materials with the largest void fractions, $\mathrm{DC}(65,5.8)$ is always larger than $\operatorname{DC}(35,1)$ because $\operatorname{DC}(65,5.8)$ conditions have a greater pressure swing of 59.2 bar in comparison to 34 bar for $\mathrm{DC}(35,1)$, enabling greater exploitation of the free volume. Materials with the smallest void fractions also tend to have a low deliverable capacity, as they exhibit less free volume for methane to occupy. In contrast to the materials with the largest void fractions, for these materials $\operatorname{DC}(35,1)$ is larger, which reflects the fact that methane tends to be relatively strongly bound in smaller pore sizes and hence favors a lower desorption pressure to reduce the methane retained in the adsorbent at depletion. For the intermediate void fractions, the relationship between $\mathrm{DC}(35,1)$ and $\mathrm{DC}(65,5.8)$ takes the form of a broad arc connecting the two extremes. Our model in Figure 4 reproduces this arc-like shape in Figure 5, as the void fraction increases monotonically with the pore size in our model (see Figure S4 in the SI). The arc can also be reproduced in a simple model by varying the Langmuirian constant $K$, which is related to the pressure at which the isotherms begin to saturate; for a fixed saturation loading, the pressure at which the isotherms saturate determines whether $\operatorname{DC}(65,5.8)$ or $\mathrm{DC}(35,1)$ is higher (see Figure S5 in the SI).

Figure 5 also shows the DOE target of $180 \mathrm{~cm}_{\text {STP }}^{3}\left(\mathrm{CH}_{4}\right) /$ $\mathrm{cm}^{3}$. We see that our arc is approaching the target in both pressure ranges but not exceeding it. However, we do see three outliers that exhibit $\mathrm{DC}(35,1)$ greater than $180 \mathrm{~cm}_{\text {STP }}^{3}\left(\mathrm{CH}_{4}\right)$ / $\mathrm{cm}^{3}$. The scarcity of materials which exceed this target (three out of 17,846$)$ illustrates a key result of our screening: it takes an outlying material with specific properties to achieve a very high deliverable capacity.

It is interesting to see how the performance of PPNs correlates with the parameters that characterize our data set. Our simple PPN model (see section 3.2. Simple Model Calculations) shows that an important parameter is the diameter of the pores. In our database, the pores are far more complex than simple spheres, but we can simply characterize each material by the size of its largest pore (denoted $D_{\mathrm{i}}$, i.e. the diameter of the largest included sphere ${ }^{35}$ ). Figure 6 illustrates $\mathrm{DC}(35,1)$ and $\mathrm{DC}(65,5.8)$ as a function of $D_{\mathrm{i}}$ for all PPNs. The qualitative behavior is very similar to that which we observed for our model PPN. For DC $(65,5.8)$, the optimal pore diameter is approximately $10 \AA$ and for $\operatorname{DC}(35,1)$ slightly lower, around $7 \AA$. Figure 6 is color-coded by VSA, which illustrates that within materials of a particular pore size, increasing surface area typically improves deliverable capacity. Figure S6 in the SI reveals how the interpenetrated and noninterpenetrated materials within the database behave differently with respect to optimum pore size. Non-interpenetrated materials exhibit pores of approximately equal size in a diamondoid arrangement. For a given material, as the level of interpenetration increases, its structure begins no longer to resemble a diamondoid network and instead transitions toward a dense, one-dimensional channel system. Hence, at large pore diameters, interpenetrated materials typically achieve higher deliverable capacities than non-interpenetrated materials due to the additional attractive forces from the atoms of the framework. This can also be observed in the higher volumetric surface area of these materials (see Figure S7 in the SI).

Here we introduce an alternative pore descriptor, the van der Waals void fraction (referred to as WVF). ${ }^{53}$ Where void fraction encodes the proportion of volume in a material that is void space, WVF encodes the proportion of volume which lies within a specific favorable interaction distance of the framework surface; intuitively, the more pore volume which enables attractive methane-framework interactions, the greater the volumetric uptake. To compute WVF, we assume that the favorable guest-framework interaction distance is in the range of 3.75 to $4.6 \AA$ (the well of the LJ potential for methane and carbon). ${ }^{53}$ In materials with large pores, the attractive regions around each internal surface of the material do not overlap, and so WVF correlates strongly with VSA (Figure S8 in the SI). However, as pore size decreases, these attractive regions begin to overlap, causing stronger methane-framework interactions but leading to a smaller fraction of the material's volume which is within the attractive region, before finally, as pore size becomes too small, both VSA and WVF tend toward zero (Figure S8 in the SI). We introduce this new descriptor because it has a noticeably different consequence for $\mathrm{DC}(65,5.8)$ compared to $\operatorname{DC}(35,1)$ : Figure S8 in the SI illustrates that, while the highest $\mathrm{DC}(35,1)$ materials exhibit a high VSA and a range of WVF, the highest $\mathrm{DC}(65,5.8)$ materials exhibit a high WVF. We postulate that these relatively simple geometric descriptors can be used to quickly rank or score materials, and we can explain these differing trends as follows.

Because of the low desorption pressure in the $\operatorname{DC}(35,1)$ metric, less residual methane is left within a material upon desorption; thus, compared to the $\mathrm{DC}(65,5.8)$ metric, it is preferential to have stronger methane-framework interactions to recruit more methane at the charging pressure. These are 
achieved by overlapping attractive potentials between different surfaces of the material, which arise from small pores; this phenomenon is captured by a high VSA, whereas WVF is unimportant. Conversely, a very high methane affinity should be avoided for a high $\operatorname{DC}(65,5.8)$ to mitigate the retention of gas at the higher discharge pressure; thus, the optimum scenario comprises nonoverlapping potentials, and hence, a maximized WVF.

The relationship between the WVF and the overall void fraction (VF) is illustrated in Figure S9 in the SI: since WVF is a subset of VF, it is never the larger of the two. As pore size increases and attractive potential regions become far apart, nonattractive regions of volume appear, leading to an optimal pore size with respect to WVF, which we note lies approximately at the optimal pore size for $\operatorname{DC}(65,5.8)$.

The heat of adsorption is an important thermodynamic property for methane storage because it measures the affinity of adsorbed methane in the material. To yield the highest deliverable capacity, the heat of adsorption must be high enough to recruit a large amount of methane at the charging pressure but low enough to release it at the discharge pressure. $^{49,52}$ Indeed, Figure 7 shows that the PPNs with the highest deliverable capacity (color-coding in Figure 7) have an intermediate heat of adsorption. In our simple model introduced above, the pore size determines the heat of adsorption. We see in Figure 7 that, to a large extent, the pore size in PPNs determines the heat of adsorption as well.

We can gain additional insight from the heat of adsorption by noting that attractive methane-methane interactions cause the heat of adsorption to increase with loading. The presence of attractive methane-methane interactions gives rise to a higher deliverable capacity than could be achieved in the absence of these interactions. ${ }^{49}$ Figure 8 displays the heat of adsorption at the charging pressure against that at the discharge pressure. Again, we see that structures with the highest deliverable capacities (color-coding in Figure 8) occur at an intermediate heat of adsorption. Further, the best materials occur above the diagonal, where the heat of adsorption is higher at the charging pressure than the discharge pressure. The reason the heat of adsorption increases with pressure, and thus loading, in these top-performing materials is the presence of attractive methanemethane interactions. These in turn recruit more methane into the framework at the tank charging pressure, but not at the discharge pressure, as the absence of adsorbed methane does not provide the extra attraction for recruitment.

We conclude that, from a design standpoint, once an optimal pore size has been achieved, one can then subsequently modify the structural chemistry to fine-tune the heat of adsorption and thus the adsorption performance (subject, of course, to changes in pore size which arise through chemical substitution). However, we did not observe any particular optimum framework chemistry which serves to enhance deliverable capacity for materials of arbitrary pore size; we did not find any significant correlations between deliverable capacity and the presence of particular functional groups or individual atoms in the framework (see Figure S10 in the SI, which presents comparisons of materials based on the presence of $\mathrm{NH}_{2}$ groups, $\mathrm{CH}_{3}$ groups, sulfur atoms, or the $A$ component of the monomer), as may be expected considering the weakness of guest-framework interactions for methane (as compared to those for polar molecules, e.g. $\mathrm{CO}_{2}$, which is well-known to interact strongly with e.g. amine functional groups within porous materials ${ }^{54-57}$ ).
Thus far we have only discussed volumetric deliverable capacity. However, high gravimetric deliverable capacity is also required to avoid massive vehicular fuel tanks, as reflected by the ARPA-E gravimetric deliverable capacity target of at least $0.5 \mathrm{~g}\left(\mathrm{CH}_{4}\right) / \mathrm{g}$ (sorbent). However, volumetric and gravimetric adsorption capacities are to a degree competing objectives (see Figure 9); design strategies to achieve materials exhibiting an optimal compromise between volumetric and gravimetric properties have recently been explored by ourselves and others. $^{26,58}$ In contrast to volumetric adsorption, any gravimetric adsorption target can be trivially exceeded, for instance, by using no sorbent at all (i.e., an infinite gravimetric uptake due to the sorbent having no mass). Hence, gravimetric deliverable capacity increases with pore size as materials converge in structure toward that of empty space. However, this of course comes at the expense of volumetric performance (and it is important to keep in mind that gravimetric adsorption capacity has a significantly lower impact on vehicle driving range than volumetric capacity ${ }^{13}$ ). It is instructive then to consider the following thought experiment: from the starting point of a free space tank, the addition of framework atoms (and hence mass) will increase the attractive guest-host forces, allowing for more methane to be adsorbed. However, after a point, additional framework atoms only occupy space, and an opportunity cost arises from the presence of framework rather than methane. This explains the peak in volumetric uptake which occurs as the gravimetric uptake decreases from that of a free space tank, demonstrating that an optimal compromise exists between volumetric and gravimetric performance.

3.3.2. Individual High Deliverable Capacity Materials. Our screening has revealed a few potentially promising PPNs for methane storage. Tables 1-4 summarize the materials with the highest $\mathrm{DC}(35,1)$ and $\mathrm{DC}(65,5.8)$, distinguished by their synthetic route. The highest $\operatorname{DC}(35,1)$ achieved was 194 $\mathrm{cm}_{\text {STP }}^{3}\left(\mathrm{CH}_{4}\right) / \mathrm{cm}^{3}$, for a germanium-based material (i.e., a PPN-5 analogue), while the highest $\mathrm{DC}(65,5.8)$ achieved was $178 \mathrm{~cm}_{\text {STP }}^{3}\left(\mathrm{CH}_{4}\right) / \mathrm{cm}^{3}$, also for a germanium-based material. In total, only three out of 17,846 materials exceeded the 180 $\mathrm{cm}^{3}{ }_{\text {STP }}\left(\mathrm{CH}_{4}\right) / \mathrm{cm}^{3}$ DOE target, and only for $\mathrm{DC}(35,1)$, which is illustrative of the degree of difficulty inherent in designing materials to exceed this target, and in turn to exceed the more recent ARPA-E target of $315 \mathrm{~cm}^{3}$ STP $\left(\mathrm{CH}_{4}\right) / \mathrm{cm}^{3}$.

We pay special attention to the three materials which exhibit $\mathrm{DC}(35,1)$ greater than $180 \mathrm{~cm}^{3}{ }_{\text {STP }}\left(\mathrm{CH}_{4}\right) / \mathrm{cm}^{3}$, denoted as PPNs A, B, and C. In the SI, ${ }^{16}$ these and all other materials are listed by their descriptive names, comprising the $A$ component name, $B$ component identifier, level of interpenetration, and a numerical identifier for disambiguating distinct conformers comprising the same $A$ and $B$ components; in this case, "hPPN Ge 3939 1-net 002", "hPPN_C 4633 1-net 001" and "hPPN__C_4080_1-net_001" respectively. Figure 10 illustrates the pore structure of these materials, along with their methane potential energy profiles and illustrations of the topological arrangement of their methane binding positions. Figure 11 illustrates the full adsorption isotherms for these materials, along with the calculated heats of adsorption as a function of loading. It is clear that these materials exhibit increasing heats of adsorption as the amount of adsorbed methane increases. Further insights can be gained from the energy landscapes and binding site positions illustrated in Figure 10. For reference in this discussion, we point to zeolite SBN ${ }^{59,60}$ which we have previously predicted to exhibit a deliverable capacity above $200 \mathrm{~cm}_{\text {STP }}^{3}\left(\mathrm{CH}_{4}\right) / \mathrm{cm}^{3}$ (in its all- 
Table 2. Six Highest DC $(35,1)$ Materials Identified for the One-Monomer Synthesis Route

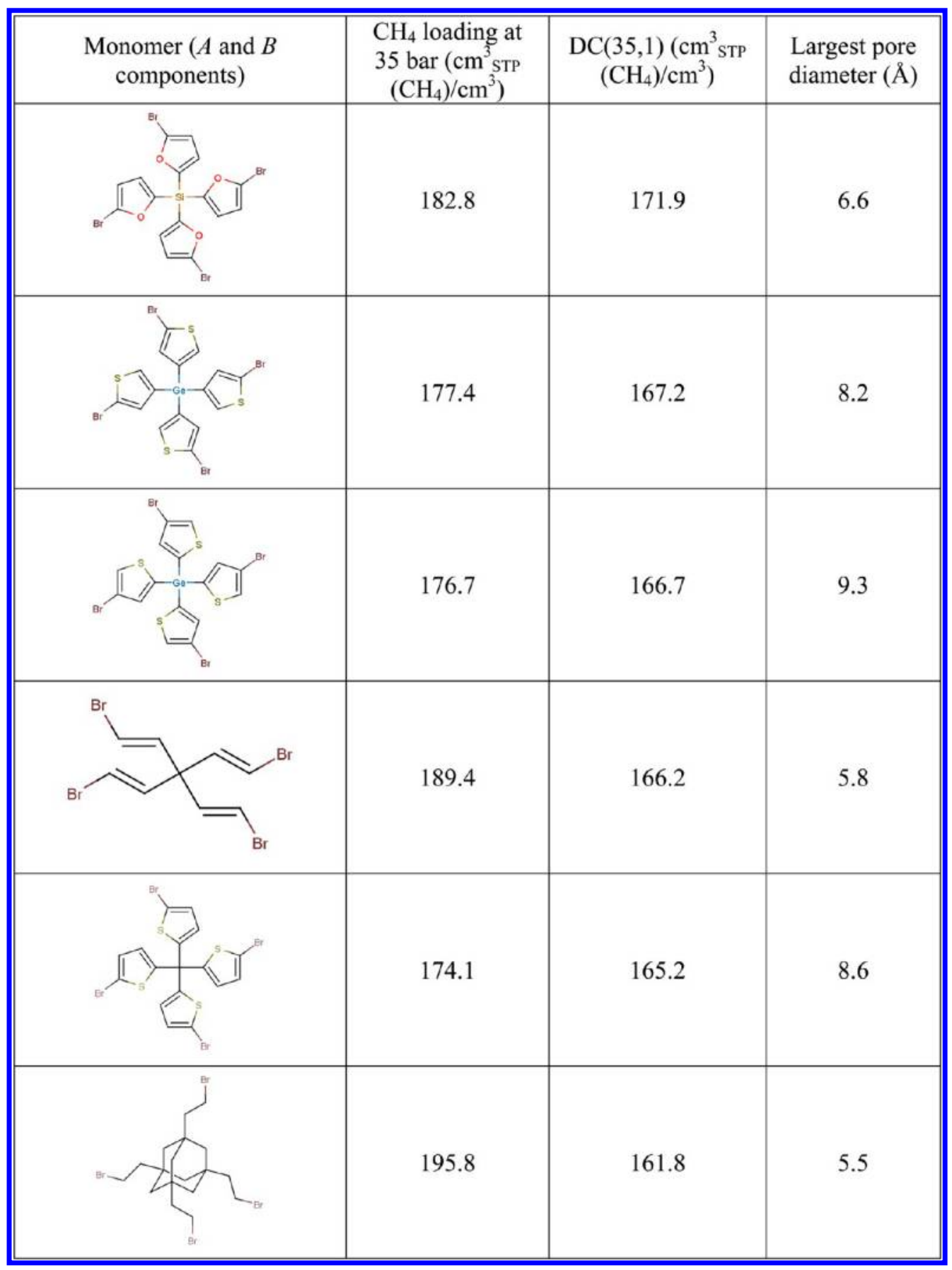

silicious form $) ;{ }^{49,61}$ this high performance is largely due to the very high density of methane binding sites $\left(6.6 \times 10^{-3}\right.$ per $\AA^{3}$ of material), each spaced approximately $4.2 \AA$ apart-the optimal distance for favorable methane-methane interaction (defined by the LJ potential).

PPN A: Our highest performing hypothetical PPN exhibits two binding sites in a unit cell of volume $321.6 \AA^{3}$ (i.e., $6.2 \times$ $10^{-3}$ per $\AA^{3}$ ), hence, a slightly lower site density than zeolite SBN. The sites are positioned at approximately $(0.25,0.25,0.25)$ and $(0.5,0.5,0.5)$ in fractional coordinates (with respect to the nonorthogonal primitive unit cell with approximately 60/60/60 degree angles), and therefore they exhibit a dia net arrangement, with edge length of $4.8 \AA$, i.e. close to the optimum pairwise distance for methane-methane interactions. The minimum binding-site energy is $-24.55 \mathrm{~kJ} / \mathrm{mol}$. This leads to a 35 bar loading of $218.3 \mathrm{~cm}_{\text {STP }}^{3}\left(\mathrm{CH}_{4}\right) / \mathrm{cm}^{3}$ and a deliverable capacity of $193.8 \mathrm{~cm}^{3}$ STP $\left(\mathrm{CH}_{4}\right) / \mathrm{cm}^{3}$.

PPN B: Our second highest performing material has a notably different character. It exhibits four sites in a much larger unit cell of $1366.8 \AA^{3}$, and so exhibits a low site density of $2.9 \times$ $10^{-3}$ sites per $\AA^{3}$, approximately half that of SBN. The minimum site energy is also weaker than our top material, at $-17.41 \mathrm{~kJ} / \mathrm{mol}$. The 35 bar loading is $210.2 \mathrm{~cm}_{\text {STP }}^{3}\left(\mathrm{CH}_{4}\right) / \mathrm{cm}^{3}$ and the deliverable capacity $189.5 \mathrm{~cm}^{3}{ }_{\text {STP }}\left(\mathrm{CH}_{4}\right) / \mathrm{cm}^{3}$. The sites exhibit a distinct geometric arrangement from that of the above, since they lie approximately at the fractional coordinates $(0,0.5,0.5) \pm(0.25,0.25,0.25)$, and so exhibit the bcu net with an edge length of $7.7 \AA$. These characteristics alone do not 
Table 3. Six Highest DC(65,5.8) Materials Identified for the Two-Monomer Synthesis Route

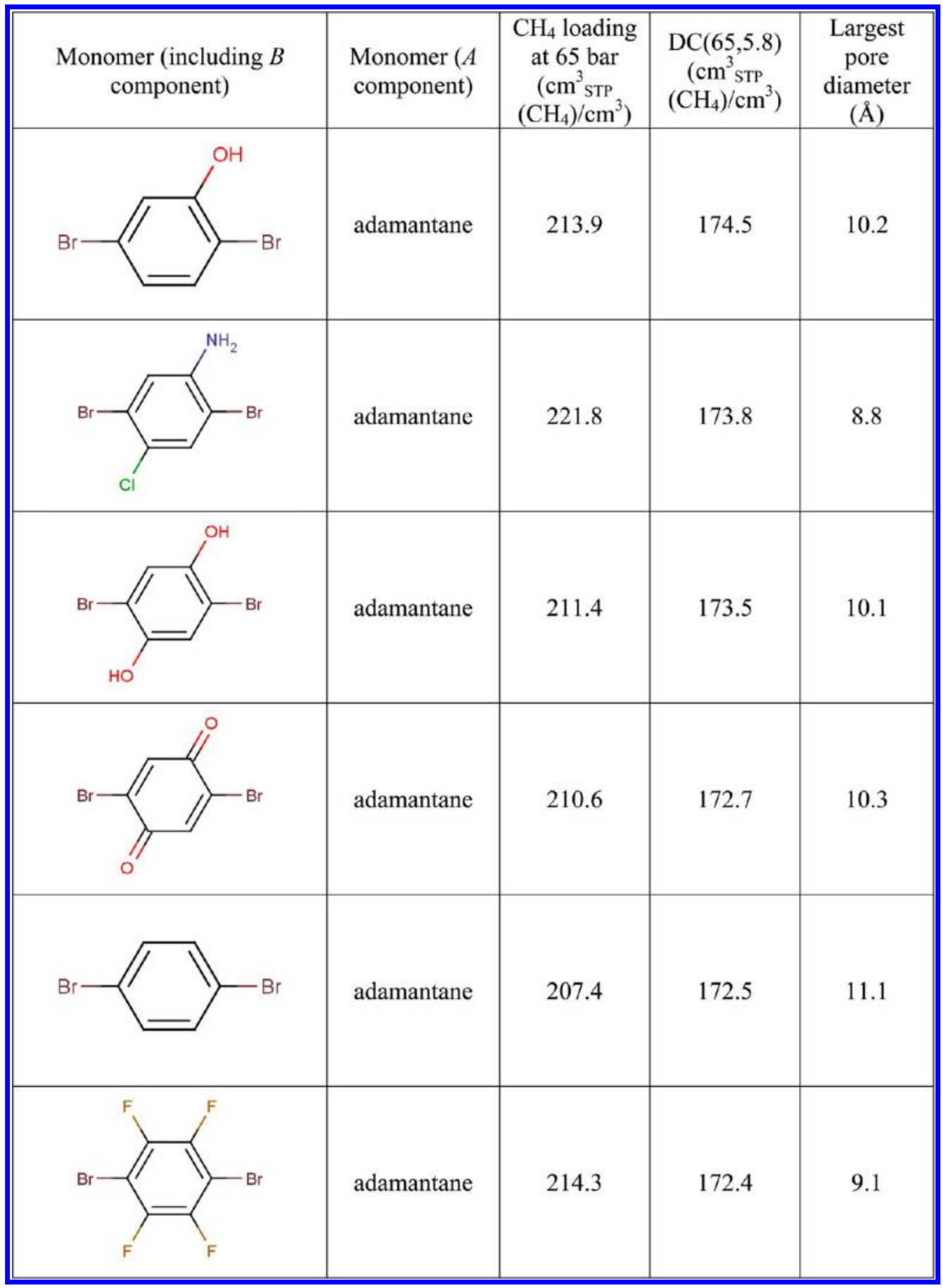

explain the high deliverable capacity of this material, and indeed during simulation we observe between 5 and 13 methane molecules per unit cell rather than the 4 which are indicated by the strongest binding sites in Figure 10; indeed, these additional methane molecules are recruited due to favorable interactions with other adsorbed methane molecules, as illustrated by the increasing heat of adsorption with increasing loading. This material's behavior clearly illustrates the benefit of favorable methane-methane interactions.

PPN C: Finally, our third best material is the extended diamond structure. ${ }^{30,62}$ It exhibits two sites in $216.8 \AA^{3}$, and hence a very high site density of $9.2 \times 10^{-3}$ per $\AA^{3}$, about $50 \%$ greater than that of zeolite SBN. These sites again exhibit the dia net, and are separated by $4.2 \AA$; hence this material should exhibit something of an 'ideal' adsorption character. Indeed, extraordinarily high loading at 35 bar of $339.0 \mathrm{~cm}_{\text {STP }}^{3}\left(\mathrm{CH}_{4}\right)$ / $\mathrm{cm}^{3}$ is observed, but the also very strong binding energy of $-27.70 \mathrm{~kJ} / \mathrm{mol}$ leads to difficulty releasing the methane at low pressure, and the deliverable capacity is significantly reduced but still very high: $185.2 \mathrm{~cm}^{3}$ STP $\left(\mathrm{CH}_{4}\right) / \mathrm{cm}^{3}$.

\section{DISCUSSION}

Our database illustrates that the materials with the highest $\mathrm{DC}(65,5.8)$ typically exhibit distinct framework chemistry to the best $\operatorname{DC}(35,1)$ structures. This is intuitive since the $\mathrm{DC}(65,5.8)$ range (higher adsorption and desorption pres- 
Table 4. Six Highest DC(65,5.8) Materials Identified for the One-Monomer Synthesis Route

\begin{tabular}{|c|c|c|c|}
\hline $\begin{array}{l}\text { Monomer ( } A \text { and } B \\
\text { components) }\end{array}$ & $\begin{array}{c}\mathrm{CH}_{4} \text { loading at } \\
65 \text { bar }\left(\mathrm{cm}^{3}{ }_{\text {STP }}\right. \\
\left.\left(\mathrm{CH}_{4}\right) / \mathrm{cm}^{3}\right)\end{array}$ & $\begin{array}{c}\mathrm{DC}(65,5.8)\left(\mathrm{cm}^{3}{ }_{\mathrm{STP}}\right. \\
\left.\left(\mathrm{CH}_{4}\right) / \mathrm{cm}^{3}\right)\end{array}$ & $\begin{array}{l}\text { Largest pore } \\
\text { diameter }(\AA)\end{array}$ \\
\hline & 203.1 & 177.5 & 10.5 \\
\hline${ }_{\mathrm{B} r}={ }_{\mathrm{si}}$ & 199.8 & 174.7 & 10.5 \\
\hline & 205.1 & 172.2 & 11.0 \\
\hline & 204.1 & 171.8 & 10.7 \\
\hline & 206.6 & 171.0 & 10.8 \\
\hline${ }^{\mathrm{x}}=$ & 207.0 & 170.8 & 9.2 \\
\hline & & & \\
\hline
\end{tabular}

sures) will favor materials which saturate at higher pressures; by contrast, the $\mathrm{DC}(35,1)$ metric favors lower pressure saturation. The best two monomer route materials for DC $(65,5.8)$ (Table 3 ) uniformly comprise single aromatic ring $B$ components and adamantane $A$ components, leading to a near-consistent pore size. In the one monomer route (Table 4), single-atom $A$ components dominate, separated by either two aromatic rings or two alkyne fragments. However, there is a degree of overlap between optimal materials across the two pressure ranges. Two structures comprising aromatic, sulfur-containing rings exist in both lists of best structures for the one-monomer route, and in general, sulfur-containing $B$ components are common in the best performing materials, as are heteroatoms in general, illustrating that, while there is no single optimum framework chemistry, chemistry can be tuned to improve performance. The best performing $\mathrm{DC}(35,1)$ PPNs typically exhibit $B$ components comprising two fused rings, or single alkyne (or double bond) fragments. The top five structures from the twomonomer route (Table 1) can be seen to produce higher deliverable capacities than the best structures from the onemonomer route (Table 2), which typically comprise two aromatic rings separated by a single bond, and hence larger pores. The case of structures exhibiting single alkyne fragments has been examined with a higher level of theory in a recent publication; $^{30}$ although these structures exhibit very high uptake at high pressures, due to their tight pores, their lowpressure uptake remains high, reducing their deliverable capacity. This is observed in the third best $\operatorname{DC}(35,1)$ structure 
(Table 1), which is identical to the extended diamond structure. ${ }^{30,62}$ The fourth best $\operatorname{DC}(35,1)$ structure exhibits a $B$ component comprising a single aromatic ring, which would lead to smaller pores-however, the use of the adamantane $A$ component in this structure leads to a counterbalancing increase in pore size. Unfortunately, materials with very small pores will suffer from diffusion limitations; indeed, the diffusion of methane within the extended diamond structure (third best material in Table 1) was reported to be very low. ${ }^{30}$

\section{CONCLUSION}

We have presented the first database of hypothetical porous polymer networks (PPNs), a class of advanced porous material consisting only of organic material. Our database of $\sim 18,000$ materials has been designed in silico from commercially available chemical fragments and via two known synthetic routes to obtain synthetically viable hypothetical materials. Each structure was relaxed using semiempirical methods to ensure structural accuracy. A unique feature of our in silico design is that we have explored the possibility of interpenetration in each structure. All of our materials were evaluated using Grand-canonical Monte Carlo molecular simulations for use as methane sorbents for vehicular fuel tanks with reference to established ARPA-E (U.S. Department of Energy) targets.

In the process of data-mining, we uncovered striking structure-property relationships that provide insight into material performance and experimental design criteria for achieving further improved materials. First, we observed that in general, the pore size is the primary geometric determinant of methane sorption performance in PPNs with the dia topology, although it is not sufficient to guarantee a high deliverable capacity (Figure 6). Our simple spherical shell model of PPNs provides insight behind the strong correlation between deliverable capacity and pore size: the pore size dictates both the free volume available for methane occupancy and the proximity of framework atoms to the adsorbed methane, and hence, the energetics of adsorption (Figure 4). Second, Figure 5 illustrates that there is a striking relationship between deliverable capacity of methane in the two considered pressure ranges. The spherical model in Figure 4 unearths this same relationship (see Figure 5, right). This 'performance arc' illustrates the difficulty in exceeding the methane storage targets, but serves to highlight that high-performing outliers may be achievable. These outliers arise from the favorable influence of methane-methane interaction within the material structure, indicated by an increase in the heat of adsorption with higher methane loadings (Figure 8). Therefore a key conclusion, which has been discussed in our recent work, ${ }^{49}$ is that these methane-methane attractions must be present in order to achieve a material with the outstanding performance required to meet and exceed the ARPA-E targets. To achieve this behavior, methane adsorption sites must be located such that the methane-methane interactions are optimal (sites approximately $4.2 \AA$ apart). In terms of experimental materials design strategy, it will not be sufficient to optimize the methane-framework interaction alone.

\section{ASSOCIATED CONTENT}

\section{S Supporting Information}

Comparison of simulated and experimental isotherms; additional plots showing correlations between various descriptors.
This material is available free of charge via the Internet at http://pubs.acs.org.

\section{AUTHOR INFORMATION}

\section{Corresponding Author}

mharanczyk@lbl.gov

\section{Notes}

The authors declare no competing financial interest.

\section{ACKNOWLEDGMENTS}

The authors thank Dr. James Stewart for providing the MOPAC license. The authors were supported by ARPA-E MOVE program under Award Number DE-AR0000249. A portion of this work was performed at the Lawrence Berkeley National Laboratory, which is supported by the Office of Science, Office of Basic Energy Sciences, of the U.S. Department of Energy under Contract No. DE-AC0205CH11231. This research used resources of the National Energy Research Scientific Computing Center, which is supported by the Office of Science of the U.S. Department of Energy under Contract No. DE-AC02-05CH11231.

\section{REFERENCES}

(1) Makal, T. A.; Li, J.-R.; Lu, W.; Zhou, H.-C. Chem. Soc. Rev. 2012, 41, 7761-7779.

(2) Lu, W.; Yuan, D.; Zhao, D.; Schilling, C. I.; Plietzsch, O.; Muller, T.; Brase, S.; Guenther, J.; Blumel, J.; Krishna, R.; Li, Z.; Zhou, H.-C. Chem. Mater. 2010, 22, 5964-5972.

(3) Yuan, D.; Lu, W.; Zhao, D.; Zhou, H.-C. Adv. Mater. 2011, 23, $3723-3725$.

(4) Lu, W.; Sculley, J. P.; Yuan, D.; Krishna, R; Wei, Z.; Zhou, H.-C. Angew. Chem. Int. Ed. 2012, 51, 7480-7484.

(5) Lu, W.; Yuan, D.; Sculley, J. P.; Zhao, D.; Krishna, R.; Zhou, H.C. I. Am. Chem. Soc. 2011, 133, 18126-18129.

(6) Ben, T.; Ren, H.; Ma, S. Q.; Cao, D. P.; Lan, J. H.; Jing, X. F.; Wang, W. C.; Xu, J.; Deng, F.; Simmons, J. M.; Qiu, S.; Zhu, G. Angew. Chem. Int. Ed. 2009, 48, 9457-9460.

(7) Trewin, A.; Cooper, A. I. Angew. Chem. Int. Ed. 2010, 49, 15331535.

(8) Martin, R. L.; Niknam Shahrak, M.; Swisher, J. A.; Simon, C. M.; Sculley, J. P.; Zhou, H.-C.; Smit, B.; Haranczyk, M. J. Phys. Chem. C 2013, 117, 20037-20042.

(9) O’Keeffe, M.; Peskov, M. A.; Ramsden, S. J.; Yaghi, O. M. Acc. Chem. Res. 2008, 41, 1782-1789.

(10) Wilmer, C. E.; Leaf, M.; Lee, C. Y.; Farha, O. K.; Hauser, B. G.; Hupp, J. T.; Snurr, R. Q. Nat. Chem. 2012, 4, 83-89.

(11) Deem, M. W.; Pophale, R.; Cheeseman, P. A.; Earl, D. J. J. Phys. Chem. C 2009, 113, 21353-21360.

(12) Pophale, R.; Cheeseman, P. A.; Deem, M. W. Phys. Chem. Chem. Phys. 2011, 13, 12407-12412.

(13) Mason, J. A.; Veenstra, M.; Long, J. R. Chem. Sci. 2014, 5, 3251.

(14) Celzard, A.; Fierro, V. Energy Fuels 2005, 19, 573-583.

(15) International Energy Agency, World Energy Outlook 2011: Are We Entering a Golden Age of Gas, www.worldenergyoutlook.org.

(16) http://www.nanoporousmaterials.org/databases/ (accessed Feb 28th 2014).

(17) Rose, M.; Böhlmann, W.; Sabo, M.; Kaskel, S. Chem. Commun. 2008, 21, 2462-2464.

(18) Fournier, J.-H.; Wang, X.; Wuest, J. D. Can. J. Chem. 2003, 81, 376-380.

(19) Ben, T.; Ren, H.; Ma, S. Q.; Cao, D. P.; Lan, J. H.; Jing, X. F.; Wang, W. C.; Xu, J.; Deng, F.; Simmons, J. M.; Qiu, S. L.; Zhu, G. S. Angew. Chem. Int. Ed. 2009, 48, 9457-9460.

(20) http://www.emolecules.com (accessed 10/02/12). 
(21) Martin, R. L.; Lin, L.-C.; Jariwala, K.; Smit, B.; Haranczyk, M. J. Phvs. Chem. C 2013, 117, 12159-12167.

(22) Ockwig, N. W.; Delgado-Friedrichs, O.; O'Keeffe, M.; Yaghi, O. M. Acc. Chem. Res. 2005, 38, 176-182.

(23) Alexandrov, E. V.; Blatov, V. A.; Kochetkov, A. V.; Proserpio, D. M. CrustEngComm 2011, 13, 3947-3958.

(24) Martin, R. L.; Haranczyk, M. Cryst. Growth Des. 2014, DOI: $10.1021 / \operatorname{cg} 500158$ c.

(25) O'Keeffe, M.; Yaghi, O. M. Chem. Rev. 2012, 112, 675-702.

(26) Martin, R. L.; Haranczyk, M. Cryst. Growth Des. 2013, 13, $4208-4212$.

(27) Stewart, J. J. P. L. Mol. Model. 2007, 13, 1173-1213.

(28) Korth, M.; Pitoňák, M.; Rezáč, J.; Hobza, P. J. Chem. Theory Comput. 2010, 6, 344-352.

(29) Řezáč, J.; Fanfrlik, J.; Salahub, D.; Hobza, P. J. Chem. Theory Comput. 2009, 5, 1749-1760.

(30) Haranczyk, M.; Lin, L.-C.; Lee, K.; Martin, R. L.; Neaton, J. B.; Smit, B. Phys. Chem. Chem. Phvs. 2013, 15, 20937-20942.

(31) Batten, S. R. CrustEnoComm 2001, 3, 67-72.

(32) Shekhah, O.; Wang, H.; Paradinas, M.; Ocal, C.; Schupbach, B.; Terfort, A.; Zacher, D.; Fischer, R. A.; Woll, C. Nat. Mater. 2009, 8, 481-484.

(33) Zhang, J.; Wojtas, L.; Larsen, R. W.; Eddaoudi, M.; Zaworotko, M. J. I. Am. Chem. Soc. 2009, 131, 17040-17041.

(34) Uribe-Romo, F. J.; Hunt, J. R.; Furukawa, H.; Klöck, C.; O'Keeffe, M.; Yaghi, O. M. I. Am. Chem. Soc. 2009, 131, 4570-4571.

(35) Willems, T. F.; Rycroft, C. H.; Kazi, M.; Meza, J. C.; Haranczyk, M. Microporous Mesoporous Mater. 2012, 149, 134-141.

(36) Bondi, A. I. Phvs. Chem. 1964, 68, 441-452.

(37) Rowland, R. S.; Taylor, R. I. Phvs. Chem. 1996, 100, 7384-7391.

(38) Allen, F. H. Acta Crystallogr., Sect. B 2002, 58, 380-388.

(39) U.S. Department of Energy DE-FOA-0000672, "Methane Opportunities For Vehicular Energy (MOVE)"; see https://arpa-efoa.energy.gov.

(40) Frenkel, D.; Smit, B. Understanding Molecular Simulations: From Algorithms to Applications, 2nd ed.; Academic Press, New York, 2002).

(41) Mayo, S. L.; Olafson, B. D.; Goddard, W. A. J. Phys. Chem. 1990, 94, 8897-8909.

(42) Martin, M. G.; Siepmann, J. I. J. Phys. Chem. B. 1998, 102, 2569-2577.

(43) Chen, T.; Chidambaram, M.; Liu, Z.; Smit, B.; Bell, A. T. J. Phys. Chem. B. 2010, 114, 5790-5794.

(44) Peng, D.-Y.; Robinson, D. B. Ind. Eng. Chem. Fundam. 1976, 15, $59-64$.

(45) Cristancho, D. E.; Mantilla, I. D.; Ejaz, S.; Hall, K. R. J. Chem. Eno. Data 2010, 55, 826-829.

(46) Zhang, M.; Perry, Z.; Park, J.; Zhou, H.-C. Polymer 2014, 55, 335-339.

(47) Pophale, R.; Daeyaert, F.; Deem, M. W. J. Mater. Chem. A 2013, 1, 6750-6760.

(48) Konstas, K.; Taylor, J. W.; Thornton, A. W.; Doherty, C. M.; Lim, W. X.; Bastow, T. J.; Kennedy, D. F.; Wood, C. D.; Cox, B. J.; Hill, J. M.; Hill, A. J.; Hill, M. R. Angew. Chem., Int. Ed. 2012, 51, 6639-6642.

(49) Simon, C. M.; Kim, J.; Lin, L.-C.; Martin, R. L.; Haranczyk, M.; Smit, B. Phvs. Chem. Chem. Phvs. 2014, 16, 5499-5513.

(50) Cox, B.; Thamwattana, N.; Hill, J. Proc. R. Soc. London, Ser. A 2007, 463, 477-494.

(51) Ripmeester, J.; Ratcliffe, C. I. Phvs. Chem. 1990, 94, 7652-7656.

(52) Bhatia, S. K.; Myers, A. L. Langmuir 2006, 22, 1688-1700.

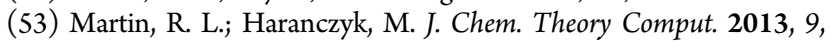
2816-2825.

(54) Demessence, A.; D’Alessandro, D. M.; Foo, M. L.; Long, J. R. J. Am. Chem. Soc. 2009, 131, 8784-8786.

(55) McDonald, T. M.; D’Alessandro, D. M.; Krishna, R.; Long, J. R. Chem. Sci. 2011, 2, 2022-2028.

(56) McDonald, T. M.; Lee, W. R.; Mason, J. A.; Wiers, B. M.; Hong,

C. S.; Long, J. R. I. Am. Chem. Soc. 2012, 134, 7056-7065.
(57) Planas, N.; Dzubak, A. L.; Poloni, R.; Lin, L.-C.; McManus, A.; McDonald, T. M.; Neaton, J. B.; Long, J. R.; Smit, B.; Gagliardi, L. $\underline{\text {. }}$ Am. Chem. Soc. 2013, 135, 7402-7405.

(58) Goldsmith, J.; Wong-Foy, A. G.; Cafarella, M. J.; Siegel, D. I. Chem. Mater. 2013, 25, 3373-3382.

(59) Bu, X. H.; Feng, P. Y.; Stucky, G. D. Nature 1998, 120, 1120411205.

(60) Shi, L.; Christensen, K. E.; Jansson, K.; Sun, J.; Zou, X. Chem. Mater. 2007, 19, 5973-5979.

(61) Kim, J.; Maiti, A.; Lin, L.-C.; Stolaroff, J. K.; Smit, B.; Aines, R. D. Nat. Commun. 2013, 4, 1694.

(62) Huang, L.; Xiang, Z.; Cao, D. J. Mater. Chem. A 2013, 1, 38513855. 